\title{
Individuelle Zeit, institutionelle Zeit, soziale Zeit
}

\section{Das Vertrauen in die Sicherheit der Rente und die Debatte um Kapitaldeckung und Umlage in Deutschland ${ }^{1}$}

\section{Philip Manow}

Max-Planck-Institut für Gesellschaftsforschung, Lothringer Str. 78, D-50677 Köln

Zusammenfassung: Der Streit um Kapitaldeckung und Umlageverfahren beginnt mit der Bismarckschen Sozialgesetzgebung und hält bis heute an. Der Artikel zeichnet die Auseinandersetzung um die Finanzierung der gesetzlichen Rentenversicherung von den achtziger Jahren des letzten Jahrhunderts bis heute nach. Im Laufe der Zeit unterlag die vorherrschende Vorstellung, wie die ,Sicherheit' der Renten am besten zu garantieren sei, einem grundlegenden Wandel. Dies weist auf den Wandel zugrundeliegender Konzeptionen von gesellschaftlicher Ordnung und gesellschaftlicher Zeit hin. Die Wahrnehmung institutioneller Anreizstrukturen und damit auch die jeweils zeitgebundene Antwort auf die Frage, wodurch institutionelle Stabilität am besten gesichert ist, hängt wesentlich davon ab, welchen sozialen, Geltungskreis' eine Institution besitzt.

\section{Einleitung}

Aktuelle Umfragen zeigen, daß das Vertrauen in die Sicherheit von Rentenansprüchen deutlich gesunken ist. Eine vor kurzem durchgeführte Allensbach-Umfrage ergab, daß heute nur noch $16 \%$ der Befragten ihre Rente für sicher halten. Im Vergleich hierzu waren es selbst 1977 - also kurz nach dem aufwühlenden Wahlkampf von 1976, in dem die sogenannte Rentenlüge eine so prominente Rolle gespielt hatte, - noch immerhin über $34 \%$ (1979 dann bereits schon wieder 52\%; siehe Köcher 1997).

Wenn man sich der Frage nähern möchte, was die Vorstellung von Sicherheit in diesem Kontext be-

${ }^{1}$ Dank für hilfreiche Hinweise an Florian Tennstedt und Ulrike Haerendel. Für hilfreiche Kritik und Kommentierungen bin ich außerdem Philipp Genschel, Hans-Willy Hohn und Eric Seils dankbar. Eine frühere Version dieses Papiers wurde in der Kolloquiumsreihe des Instituts für Sozialpolitik der Universität Göttingen und an der Universität Gesamthochschule Kassel, sozialpolitisches Kolloquium, vorgestellt. Vielen Dank für die Gelegenheit zur Präsentation und für die kritische Diskussion. Sehr hilfreich war Annette Vogel beim Korrekturlesen, dem Erstellen der Grafiken und der allgemeinen Fehlerbeseitigung. Herr Mörschel vom Verband Deutscher Rentenversicherungsträger (VDR) und Herr Genske von der Bundesversicherungsanstalt für Angestellte (BfA) waren mit Angaben zu der Vermögenslage der Arbeiter- und Angestelltenversicherung behilflich. Auch hierfür vielen Dank. deuten kann, läßt sich eine Beobachtung Franz Xaver Kaufmanns als analytischer Ausgangs- und Bezugspunkt nehmen. Kaufmann bemerkt, „daß es in der Idee der Sicherheit stets um die ,Vernichtung der Zeitlichkeit der Zukunft" geht" (Kaufmann 1973: 157; Hervorhebung des Originals weggelassen). Diese Beobachtung scheint in besonderem Maße für den Strang der sozialen Sicherheit zuzutreffen, der gegen das ,Altersrisiko“ versichert, also in Deutschland für die gesetzliche oder soziale Rentenversicherung. Hierbei spielen wenn man bei Kaufmanns Begrifflichkeit bleiben will - zunächst zwei Zeiten eine Rolle, die individuelle Zeit und institutionelle Zeit genannt werden können. Die Altersversicherung ist eine Institution, deren Funktion darin besteht, in der Zukunft liegende individuelle (Einkommens-)Unsicherheit in ein kalkulierbares Risiko zu verwandeln. Dafür wird in der Gegenwart eine Prämie erhoben. Diese Prämie kann als materialisierter Ausdruck einer gelungenen Vernichtung der Zeitlichkeit der $\mathrm{Zu}$ kunft verstanden werden, insofern sie ,angemessen' oder hinreichend zur Deckung des Risikos erscheint und insofern, als die Einlösung des mit der Prämie erworbenen Anspruchs auf Risikodeckung in der Zukunft selbst gesichèrt erscheint.

Die Institution einer Altersversicherung unterliegt nun einer eigenen, mit den individuellen Lebensverläufen nicht notwendigerweise deckungsgleichen Zeit. Damit ist vor allem gemeint, daß die 
„Bewegungsgesetze' dieser Institution von Akteuren mitbestimmt werden, deren Handlungskalküle und -motive nicht ausschlieBlich auf diese eigentliche Institutionenfunktion: ,Vernichtung der Zeitlichkeit der Zukunft', gerichtet sind, also nicht restlos in der Erfüllung dieser Funktion aufgehen - und das heißt vor allem: die in einem anderen Zeitrahmen kalkulieren. ${ }^{2}$ Dies zielt zunächst auf den ,short-termism' demokratischer Politik (Majone 1996; Moe 1990; Przeworski 1988, 1991). Demokratie als ein System der „organized uncertainty" und "ruled open-endedness" (Przeworski 1991: 13) scheint wenig geeignet, zur Vernichtung der Zeitlichkeit der Zukunft einen bedeutsamen Beitrag zu leisten. Nimmt man das Problem unvollständiger Information und der erhöhten Wahrscheinlichkeit der ,dynamischen Inkonsistenz' von sehr langfristigen Verträgen hinzu, so erscheint die Synchronisierung institutioneller Zeit mit individueller Zeit in der Rentenversicherung extrem voraussetzungsvoll. Die immer wiederkehrenden Vorschläge zur weitgehenden Herausnahme der Rentenversicherung aus dem politischen Entscheidungsbereich beziehen aus diesem Sachverhalt ihre Plausibilität. ${ }^{3}$

2 Zwei mehr als hundert Jahre auseinanderliegende Zitate zur politischen Rationalität sozialpolitischer Reformen mögen hier als anekdotischer Beleg für die häufig anzutreffende Inkongruenz zwischen politischen und ,funktionalen' Zeithorizonten dienen: „Jeder Tag hat seine eigenen Sorgen, und es ist nicht weise, die Sorgen der Zukunft freiwillig auf die Gegenwart zu übernehmen" (dieses Bismarck-Zitat ist der Titel eines Aufsatzes von Tennstedt/ Winter [1995]). Gut hundert Jahre später erklärte David Stockman, Budget Director der Reagan-Regierung, zur Notwendigkeit einer Rentenreform angesichts der defizitären Haushaltslage der Social Security Administration: "[I have no interest in wasting] a lot of political capital on some other guy's problem in 2010" (zitiert nach Pierson 1997: 24).

${ }^{3}$,Zeit' ist eine selten systematisch analysierte Dimension politischen Handelns. Dort, wo die Zeitdimension der Politik einmal zum Gegenstand der Betrachtung gemacht wird, herrscht meist ein skeptisches Urteil hinsichtlich der Fähigkeit vor, längere Zeithorizonte in das politische Kalkül einzubeziehen: „Not only is monitoring often exceptionally difficult in politics, but it is also hard to hold actors accountable because of the relatively rapid turnover of key positions.(...) In many cases, the long term is essentially beyond the political horizon" (Pierson 1997: 25). Das theoretische Interesse an der zeitlichen Dimension politischer Prozesse nimmt aber offensichtlich zu. In einer Reihe neuerer Arbeiten wird z.T. methodisch (Bartolini 1993), überwiegend jedoch theoretisch an das Thema herangegangen (in diesem Zusammenhang sind insbesondere die neueren Arbeiten von Orren/ Skowronek 1994, 1995
Zugleich lenkt die Unterscheidung zwischen individueller Zeit und institutioneller Zeit die Aufmerksamkeit aber auch darauf, daß die Synchronisierung von Zeitdimensionen des Handelns zwischen Individuen und Institutionen nicht allein durch eine möglicherweise nur mangelhaft mögliche institutionelle oder politische Langfristbindung gefährdet wird, sondern auch durch einen Wandel gesellschaftlicher Zeitvorstellungen herausgefordert werden kann. Hiermit meine ich den Wandel allgemeingültiger, gesellschaftlich verbindlicher Interpretationen von Zeit, die selbst sozial und politisch geprägt sind und die sich historisch auf unterschiedliche Referenzgrößen bezogen haben (Familie: genealogische Zeit, korporative Gruppe oder Stand, Volk, Nation bzw. Staat: kollektive Zeit in verschieden breiter Abgrenzung, Individuum: biographische Zeit; vgl. Maier 1987). Eine soziale Versicherung wie die Rentenversicherung definiert ein Risikokollektiv und dieses Risikokollektiv war in Deutschland keineswegs von Beginn der Bismarckschen Sozialgesetzgebung an nationalstaatlich bestimmt. Vielmehr hat es eine bedeutende Zeit gedauert, bis tatsächlich der $\mathrm{Na}$ tionalstaat den verbindlichen Referenzrahmen für die soziale Versicherung bildete. Heute scheint diese integrative Funktion des nationalen Sozialstaats erneut in Gefahr (Kaufmann 1997, 1997a). Wenn aber zunächst freiwillige Vereinigungen in Anlehnung an und Erweiterung von familialer oder genossenschaftlicher Selbsthilfe, dann Verteilungskoalitionen politischer Gruppen, schließlich ,das Volk' und erst nach dem zweiten Weltkrieg die Nation als sozialpolitische Bezugsgrößen fungierten (siehe unten, Abschnitte 2 bis 5), dann waren mit diesem Bezugsgrößenwandel immer auch unterschiedliche Vorstellungen verbunden über die inter-temporale Verteilungsgerechtigkeit und die institutionellen Stabilitätsbedingungen einer sozialen Versicherung gegen die Wechselfälle des Lebens - hier der Rentenversicherung.

Mein Interesse an dem Wandel von Vorstellungen von einer gerechten und praktikablen Sicherung gegen das Altersrisiko und ihrem sichtbaren, weil institutionalisierten Ausdruck in Form des Wan-

und Skowronek 1997 sowie Majone 1996 zu nennen; siehe auch die Beiträge in Elster/ Slagstad 1988 und die Überlegungen in Döhler/ Manow 1997). Nicht zufällig resultiert das Interesse von Pierson an der zeitlichen Dimension politischer Prozesse aus seiner Beschäftigung mit pension policies (vgl. Pierson 1992, 1997). Bereits sehr frühe Überlegungen zum Thema finden sich auch bei Lehmbruch (1979). 
dels der Finanzierungsverfahren in der Geschichte der GRV richtet sich auf zwei Aspekte: Zum einen kann eine historische Rekonstruktion die aktuelle Diskussion um die Vor- und Nachteile von Umlageverfahren versus Kapitaldeckungsverfahren in Perspektive setzen. Sie kann für die aktuelle Diskussion über die Sicherheit der Renten insofern erhellend sein, als sie zeigt, daß einige der heute besonders in der Kritik stehenden Elemente der gesetzlichen Rentenversicherung selbst Ausdruck eines langwierigen und oftmals schmerzhaften Lernprozesses über die langfristige Tragfähigkeit institutioneller Arrangements gewesen sind, der in der heutigen Diskussion vollständig vergessen zu sein scheint. Zum anderen und allgemeiner steht mit der Betrachtung der Auseinandersetzungen um die richtige und gerechte Finanzierung der Rente der sich über die Zeit vollziehende Wandel der ,dualen Natur' der Institution soziale Rentenversicherung im Mittelpunkt der nachfolgenden Analyse - eine Institution, die aufgrund ihrer wirtschaftlichen Bedeutung und ihres annähernd die gesamte Bevölkerung umfassenden Deckungsgrades bedeutsames Element der gesellschaftlichen Moralökonomie geworden ist. ${ }^{4}$ Der duale, sowohl instrumentelle als auch normative Charakter von Institutionen (vgl. Schimank 1992; Offe 1996) ${ }^{5}$ läßt sich an der Geschichte der Debatte um die Finanzierung der Rentenversicherung deswegen gut verfolgen, weil es in dieser Debatte zwar immer zunächst um Funktionsprobleme und Verteilungsgerechtigkeit geht, der Referenzrahmen in Hinblick darauf, für wen die Rentenversicherung funktionieren und innerhalb welcher Gruppe Verteilungsgerechtigkeit herrschen soll, aber über die Zeit hinweg wechselte. Damit steht die nachstehende Betrachtung im Schnittpunkt zweier alternativer Deutungen über die Funktion von Institutionen und den Ursachen institutionellen Wandels: Auf der einen Seite eine eher ökonomisch rationale Interpretation (,the rational choice account of trust"; Hardin 1990, 1992, 1996), die die Schwierigkeiten glaubwürdiger Langfristbindung betont und Wandel aus den Defiziten institutioneller Anreiz- und Möglichkeitsstrukturen erklärt (Dasgupta 1988; Levi 1996). Auf der anderen Seite die Betonung

\footnotetext{
${ }^{4}$ Zur Analyse der Rentenversicherung als einer Manifestation einer spezifisch modernen ,Moralökonomie‘ siehe vor allem Kohli (1989).

${ }^{5}$ Siehe die Gegenüberstellungen bei Offe (1996: 201). Institutionen müssen zugleich ermöglichen "consolidation of beliefs" und ,implementation of goals". Der doppelte Imperativ lautet: „doing things the right way" und ,getting things done" (Offe 1996: 201).
}

der ,moralökonomischen“ Dimension von gesellschaftlicher Zeit und institutioneller Ordnungen und damit der normativen Einbettung von interessengeleitetem Handeln. Daher kann die vorliegende Analyse schließlich auch insofern ertragreich sein, als sie durch die Untersuchung des Wandels sozialer Zeitvorstellungen, der Ausdruck wechselnder gesellschaftlicher Ordnungsvorstellungen ist, den historisch wechselnden Bezugsrahmen nutzenkalkulierenden Handelns aufzeigt.

Im folgenden möchte ich anhand der Geschichte der GRV-Finanzierungsverfahren diese beiden eng zusammenhängenden Themenbereiche, die institutionelle Generierung von langfristiger Erwartungssicherheit einerseits und den Wandel zugrundeliegender gesellschaftlicher Vorstellungen von Zeit und gerechter (Zeit-)Ordnung andererseits, analysieren. Ich gehe wie folgt vor: Abschnitt 2 behandelt die Auseinandersetzung um Umlage und Kapitaldeckung bei der Einführung der Unfall- und Rentenversicherung, die im wesentlichen ein Konflikt zwischen einem liberalen und ,etatistischen" Verständnis der neuen sozialen Versicherung war. Abschnitt 3 behandelt die Zwischenkriegszeit bis 1935 und befaßt sich mit der großen Herausforderung, vor die das Kapitaldeckungsverfahren durch Inflation und Weltwirtschaftskrise gestellt wurde. Der Streit um Kapitaldeckung und Umlage wurde hier als Gruppenkonflikt ausgetragen, für den es in der fragmentierten Gesellschaft der Weimarer Zeit jedoch keine Lösung gab. In Abschnitt 4 wird die beginnende Umorientierung von der Kapitaldeckung zur Umlagefinanzierung unter den Nationalsozialisten geschildert. Bezugspunkt war nun die eine, völkische Schicksalsgemeinschaft. Eine Interpretation, die von ihren völkischen Elementen bereinigt dann auch für die frühe Bundesrepublik verbindlich geworden ist. In einem Ausblick (Abschnitt 5) wird die aktuelle Debatte um die Umlagefinanzierung in der gesetzlichen Rentenversicherung kurz angesprochen, die von einem vollständigen Auseinandertreten von individueller und institutioneller Zeit gekennzeichnet zu sein scheint. Zudem wird das Geschilderte im Lichte der konkurrierenden Hypothesen über die Stabilität und Vertrauenswürdigkeit institutioneller Arrangements diskutiert.

\section{1889-1914: Liberale Zeit}

Das Umlageverfahren galt in Deutschland unter den akademischen Experten und den zuständigen Fachbürokraten lange Zeit als ein besonders unseriöses Verfahren zur Finanzierung der sozialen 
Rentenversicherung. In den parlamentarischen Beratungen zum Gesetz betreffend die Invaliditätsund Alterssicherung vom 22. Juni 1889 (RGBl. I, 97; IuAVG) machte sich die Unternehmerschaft zwar dafür stark, die Alters- und Invalidenversicherung durch das Umlageverfahren zu finanzieren, wenn schon die Einführung der Versicherung im ganzen politisch nicht abzuwenden sei (Rosenstock 1934: 64). Dies hatte in erster Linie darin seinen Grund, daß sich die Unternehmen vom Umlageverfahren zunächst nur geringe, langsam steigende und somit erst später spürbare Beitragsbelastungen erwarteten. Doch erschien dieser Vorteil des Umlageverfahrens aus der Sicht der zuständigen Ministerialreferenten gerade als Beweis seiner mangelnden Solidität und des in ihm zum Ausdruck kommenden „Leichtsinn[s]“, welcher geeignet war, „die Gegenwart auf Kosten späterer Jahrzehnte zu entlasten“ (Rosenstock 1934: 65).

Der Vorteil der Kapitaldeckung wurde dagegen in ihrer größeren, wohl auch als erzieherisch wirksam vorgestellten Transparenz gesehen. Die Kapitaldeckung offenbare "die ganze Last [...] zwar schneller, aber dann auch konstanter [...] als das Umlageverfahren, wo sich die volle Auswirkung der Belastung, d.i. der Beharrungszustand, in dem ,ein stationärer Rentenbestand mit unveränderlicher Anzahl und gleichbleibender Alterszusammensetzung einem stationären Versicherungsbestand gegenübertritt', erst nach Jahrzehnten gezeigt hätte" (Rosenstock 1934: 65; Zitat im Zitat aus Manes Versicherungslexikon, Berlin 1930). Es ging also darum, daß das Verfahren der Kapitaldeckung angeblich ehrlicher war, und nur diese größere Ehrlichkeit schien langfristiges Vertrauen in die finanzielle Solidität der Rentenversicherung rechtfertigen zu können. Die gesamte zur Anwendung gebrachte Semantik von Leichtsinn versus Solidität, vom Leben auf Kosten der Zukunft versus vernünftiger und verantwortlicher Kalkulation, von Konsum versus Sparen ist natïrlich in den Kontext jenes größeren bürgerlichen Disziplinierungsprojekts einzuordnen, in dessen Rahmen die soziale Frage im letzten Quartal des 19. Jahrhunderts verhandelt wurde.

Die bis in die 30er Jahre des 20. Jahrhunderts hinein prominente Denkfigur des ,Beharrungszustands' zeigt dabei, daß zu den Hintergrundannahmen dieser Diskussion die Vorstellung von einer linearen, teils sogar stationären Wirtschafts- und Bevölkerungsentwicklung gehörte (Thullen 1982: 129). Zeit stellte man sich als kontinuierlich und absolut vor (Maier 1987: 154-159). Für die Zukunft hieB es in vernunftiger, vorsichtiger, voraus- schauender Kalkulation täglich einen kleinen Konsumverzicht $\mathrm{zu}$ leisten und einen festen und über die Zeit konstanten Betrag ,beiseite zu legen“. In der Frage ,Umlageverfahren oder Kapitaldeckung' ging es unter der Annahme einer stationären Wirtschaft und Bevölkerung, übrigens auch unter der Annahme eines im wesentlichen gleichbleibenden Leistungsrechts, zunächst also nur um verschiedene Formen des ,phasing-in'. Die vorherrschende Vorstellung von Linearität zeigte sich auch daran, daß in der zeitgenössischen Literatur das sogenannte Anwartschaftsdeckungsverfahren mit dem Verfahren der allgemeinen Durchschnittsprämie gleichgesetzt wurde. Der Rentenbeitrag errechnete sich beim Anwartschaftsdekkungsverfahren aus der Erfordernis, daß der Gegenwartswert aller zukünftigen Beiträge zusammen mit dem aktuellen Vermögen alle gegenwärtig gezahlten Renten und alle zukünftigen Leistungsansprüche deckte. Unter der Annahme, daß sich Bevölkerung und Wirtschaft linear entwickelten und das Rentenrecht unverändert blieb, führte dies sowohl zu einer anfänglich hohen Kapitalbildung als auch zu einer für alle gleichen und über die Zeit konstanten Prämie. Die beiden wichtigen Elemente, Sicherheit der Ansprüche (durch Kapitalbildung) und gerechte Kostenverteilung (durch Beitragskonstanz), schienen somit in offensichtlicher Weise gesichert.

Auch wenn schließlich im weiteren Gesetzgebungsprozeß statt des Anwartschaftsdeckungsverfahrens ein zunächst auf zehn Jahre berechnetes Kapitaldeckungsverfahren eingeführt wurde, ${ }^{6}$ so blieb doch die Anwartschaftsdeckung ,als das prinzipielle Ziel“" (Rosin 1914: 103), sozusagen als regulative Idee der Altersversicherung, in den zuständigen Expertenkreisen weitgehend unumstritten. Heinrich Rosin, einer der führenden Sozialrechtsexperten der Zeit, meinte durch ein einfaches Gedankenexperiment aufzeigen zu können, inwiefern das Umlageverfahren im Vergleich zum System der Kapital- oder Anwartschaftsdeckung der nötigen „versicherungstechnische[n] Sicher-

${ }^{6}$ Die heutige Diskussion kennt diese Differenzierung zwischen Anwartschafts- und Kapitaldeckung zumeist nicht mehr. In der zeitgenössischen Debatte meinte Kapitaldeckung die Deckung der Anwartschaften eines bestimmten Zeitabschnitts. Das Anwartschaftsdeckungsverfahren war hingegen nicht zeitbegrenzt. Die Abkehr von der Anwartschaftsdeckung zugunsten der auf zehn Jahre berechneten Kapitaldeckung war vor allem durch $\mathrm{Be}$ fürchtungen begrindet, die Anhäufung sehr großer Kapitalien habe wirtschaftliche Nachteile und provoziere politischen Mißbrauch. 
heit entbehrt" (Rosin 1914: 96). In welchem Ausmaß - so fragte Rosin - sind durch Prämienzahlungen gewonnene Ansprüche an ein Versicherungsinstitut gesichert, „wenn man sich die Geschäftstätigkeit des Instituts am Schlusse eines Geschäftsjahres eingestellt denkt"? (Rosin 1914: 96-97). ${ }^{7}$ Die Beweisführung schien ausgesprochen einleuchtend. Allein beim Anwartschaftsdeckungsverfahren wären nicht nur die bereits bewilligten Renten gesichert, sondern darüber hinaus auch alle bereits durch die Beitragszahlungen erworbenen Leistungsansprüche (Anwartschaften), während beim Umlageverfahren, ,soweit nicht besondere Massen, insbesondere ein Reservefonds, zur Verfügung stehen, nicht einmal soviel Vermögen vorhanden [ist], um auch nur die Jahresraten späterer Jahre auf die schon bewilligten Renten zu zahlen" (Rosin 1914: 97). Beim Anwartschaftsdekkungsverfahren hingegen könne im Falle der „Einstellung des Versicherungsbetriebs“ die „aufgelöste Anstalt sowohl die Rentner als auch die Gesamtheit der Aktiven mit einem ihrem Anspruch bzw. ihrer Anwartschaft entsprechenden Kapitalbetrag abfinden.“ Rosin folgerte: „Es ist klar, daß damit die größte Sicherheit erreicht ist" (Rosin 1914: 98 und 99).

Was an Rosins Gedankenexperiment vor allem auffällt, ist einerseits die ,auf eine ewige Folge von Generationen angelegte" Berechnung des Beitragssatzes, andererseits das „systematische Bedürfnis, sich [...] die Wirkung desselben im Endlichen, d.h. unter Voraussetzung einer Einstellung der Versicherung klar zu machen" (Rosin 1914: 104 und 103; gesperrt im Original). Die argumentative Überzeugungskraft des Bezugs auf ein fiktives Ende der Versicherung kann dabei wohl kaum auf die reichhaltigen Erfahrungen der Zeitgenossen mit dem Bankrott einzelner Krankenkassen, Versicherungsunternehmen oder Banken zurückgeführt werden, denn im Fall des Bankrotts einer Landesversicherungsanstalt hätten keine Ansprüche ausgezahlt werden können. Und zugleich war es schwer einzusehen, warum man ein systematisches Bedürfnis haben sollte, sich die Wirkung des Finanzierungsmodus ,im Endlichen' zu vergegenwärtigen, wenn ein Bankrott der Alters- und Invalidenversicherung nicht eintrat. Zudem war auch die mit der Bismarckschen Sozialgesetzgebung verwirklichte öffentlich-rechtliche Sicherung ge-

\footnotetext{
${ }^{7}$ Dieses Argument geht nicht auf Rosin selber zurück, sondern war bereits bei der Debatte um die Invaliditätsund Altersversicherung prominent (siehe etwa Bosse/ Woedtke 1891).
}

gen die ,Wechselfälle des Lebens' immer mit dem vermeintlichen Vorteil einer faktisch nicht vorhandenen oder doch deutlich verringerten Insolvenzgefahr gegenüber der in dieser Hinsicht viel verletzlicheren rein privatrechtlichen Versicherungslösung begründet worden. So muß die Widersprüchlichkeit des gleichzeitigen argumentativen Bezugs auf ,Endlichkeit' und ,Ewigkeit' der neuen sozialen Versicherung vielmehr als Ausdruck einer Zwischenlage verstanden werden, in der zwar nun der Staat zunehmend in die Verantwortung für die Sicherung gegen soziale Risiken trat, die Art und Weise dieser staatlichen Verantwortungsübernahme aber noch stark von dem vorherigen privaten, bürgerlich-familialen Sicherungsmodell geprägt war beziehungsweise von dem liberalen Modell der Unterstützung auf Gegenseitigkeit.

In Rosins Argumentation spielte daher der fiktive ,Tod einer Institution' die Rolle jener kurzzeitigen Interpunktion eines in die Ewigkeit zielenden linearen Zeitpfeils (begründet auf der ,ewigen Abfolge von Generationen'), der im Kontext der bürgerlichen Familie durch den Tod des pater familias markiert war und zu dessen Zeitpunkt der Übergang des bis dahin akkumulierten familiären Reichtums an die Kinder stattfand (also die Auszahlung der durch die ,Beiträge' zur familiären Zugewinngemeinschaft erworbenen Ansprüche an die ,Beitragszahlenden'). Genau gegen diese Vorstellung des Todes einer Institution bzw. einer ,moralischen Person' hatte sich Bismarck vehement gewandt, als der Streit um Kapitaldeckung oder Umlage bereits bei der Unfallversicherung aufgebrochen war. Den Bedenken des Referenten des Reichsamts des Inneren, Theodor Lohmann, gegen das Umlageverfahren, daß nämlich mit ihm ,die entstehenden Verpflichtungen nicht von den gegenwärtig vorhandenen Beteiligten, sondern von den mit diesen vielleicht nicht identischen künftigen Mitgliedern der Genossenschaft zu tragen sein werden", setzte Bismarck knapp und bestimmt entgegen: „Der Wechsel der Individuen ist irrelevant, findet in allen staatlichen Verhältnissen statt. Die Corporation, moralische Person, ist permanent wie der Staat" (Quellensammlung 1995: Nr 44 S. 171 und Fn. 9). Die Vorstellung, daß „korporative Verbände nicht sterben" (Bismarck, zit. nach Quellensammlung 1995: Nr.26 S.93, Fn. 4), daß sich schließlich die „unbeschränkte Dauer“ der neuen Versicherung im Staat begründet, daß es nun der Staat - und nicht mehr die Familie - ist, der die „nie unterbrochene Folge versicherter Generationen“ garantiert, daß der Staat für die „péren- 
nité" ${ }^{\prime 8}$ einer „öffentlich-rechtlichen, gesetzlich verankerten obligatorischen Rentenversicherung" verbürgt (Thullen 1982: 126), war - zumindest für Deutschland - eine radikale und visionäre Idee, die von der zeitgenössisch ,herrschenden Lehre von der Überlegenheit der privatversicherungsrechtlichen Kapitaldeckung wenig beeinflußt und beeindruckt war. Es war dann bezeichnenderweise auch der Streit um Kapitaldeckung oder Umlage, in dessen Zusammenhang der bekannte Bismarcksche Satz fiel: „Der Staat und seine Einrichtungen sind nur möglich, wenn sie als permanent identische Persönlichkeiten gedacht werden“ (Quellensammlung 1995: Nr. 48 S. 183).

Doch der Nationalstaat war für die Deutschen ein so neues Phänomen, und der Zeitpunkt, zu dem der Staat Verantwortung für die Versicherung der Bevölkerung gegen die sozialen Risiken übernahm, war so früh, daß gegen Bismarcks Willen privatversicherungsrechtliche, liberale Formelemente in der Gestaltung der neuen öffentlichrechtlichen Rentenversicherung noch lange Zeit prägend blieben. Hier ist insbesondere die fortdauernde legitimatorische Prägekraft des gerade sukzessive abgelösten bürgerlichen Familienmodells beziehungsweise des liberalen Modells der Unterstützungsvereine auf Gegenseitigkeit zu vermerken. Für die Unfallversicherung und auch für die Krankenversicherung wurde zwar das Umlageverfahren festgeschrieben, bei der auf längere Zeiträume berechneten Alters- und Invalidenversicherung kam hingegen zunächst das auf einen zehnjährigen Abschnitt berechnete Kapitaldekkungsverfahren zum Zuge. Bismarck selber intervenierte in den Gesetzgebungsprozeß zur Altersund Invalidenversicherung nicht mehr sonderlich. Bestimmend waren nun stärker die Referenten des Reichsamts des Inneren und die Parteien. Dadurch setzte sich eine andere Vorstellung von der Rolle des Staates in der neuen Sozialversicherung durch. Mit bezeichnender Argumentation lehnte man nun das Umlageverfahren ab: „Durch das Umlageverfahren werde allerdings die Gegenwart entlastet, aber die Belastung der Zukunft sei eine unberechenbare, und eine derartig leichtsinnige Wirtschaft dürfe sich ein bonus pater familias

${ }^{8}$ Ich vermute, es ist kein $\mathrm{Zufall,}$, daß dieser versicherungsmathematische Fachbegriff aus dem Französischen stammt, denn die Vorstellung eines sich im Rahmen des Nationalstaats formenden und sich durch ihn definierenden Risikokollektivs, dessen ,ewige ${ }^{\prime}$ Reproduktion der Staat garantiert, konnte in Frankreich wohl seine fruheste und akzentuierteste Ausprägung finden. nicht zu Schulden kommen lassen; als solchen aber müsse der Gesetzgeber sich immer betrachten" (Bosse und Woedtke 1891: 285; meine Hervorhebung).

Wenn sich das Festhalten an der Kapitaldeckung aus diesem frühen Übergang vom liberal-bürgerlichen zum staatlichen Regulierungsmodell erklärt (und eben auch aus dem besonders jungen Alter des neuen Staats), so deutet sich doch schon an, welchen Bedeutungswandel ein Begriff wie der der ,Generation“ bei diesem Übergang durchmachte und wie sich gleichsam unter der Hand die zugrundeliegende Vorstellung von Zeit schrittweise von einer genealogischen $\mathrm{zu}$ einer kollektiven Konzeption wandelte. Denn als man zehn Jahre nach Verabschiedung des IuAVG mit dem Invalidenversicherungsgesetz 1899 das Verfahren der allgemeinen Durchschnittsprämie einführte, meinte man zwar, nun dem Ideal der Anwartschaftsdeckung näher gekommen zu sein, vor allem, da der neue Kalkulationshintergrund nun tatsächlich die ,ewige Abfolge zukünftiger Generationen" war. Doch der Einwand der Versicherungsmathematik, daß zwangsläufig zwischen den Generationen umverteilt werde, wenn sich die Altersstruktur zwischen ihnen verändere (etwa durch ein geringeres Invaliditätsrisiko oder längere Erwerbsbiographien späterer Generationen), tat nun schon nichts mehr zur Sache (vgl. Rosin 1914). Faktisch hatte sich gegen das bei der Privatversicherung abgeguckte Gebot individueller (und generationaler) Beitragsäquivalenz bereits das Recht des Staats zur abstrakten Gleichbehandlung ,seiner' Bürger durchgesetzt. „Das Verfahren der allgemeinen Durchschnittsprämie kam [...] der sozialpolitischen Vorstellung entgegen, im Zeitverlauf einen konstanten Beitragssatz zur Rentenversicherung $\mathrm{zu}$ haben und damit nicht eine Generation von Beitragszahlern stärker zu belasten als die andere" (Mörschel 1990: 627). So wurde allmählich klar: „Das moralische Universum ist nicht mehr das Verwandtschaftsnetz oder [...] die lokale Gemeinschaft, sondern der neue Nationalstaat“ (Kohli 1989: 538-539). Wichtiger noch als das bei der Bismarckschen Sozialgesetzgebung im Vordergrund stehende Projekt - die Generierung von Loyalität gegenüber dem neuen Staat durch besondere sozialstaatliche Leistungsgewährung - war somit wohl, daß „das Wohlfahrtssystem [...] dazu bei[trug], die Nation als einen kollektiven Bezugsrahmen für die Identität zu konstruieren“ (Kohli 1989: 539). 


\section{1914-1935: Kollektive Zeit}

Die Überzeugung von der Überlegenheit und der größeren Seriösität des Verfahrens der Anwartschaftsdeckung hielt sich bis Mitte der 30er Jahre und wurde zwischenzeitlich nicht von der Erfahrung demographischer Wechsellagen, sondern von der Erfahrung extremer wirtschaftlicher Volatilität erschüttert. Der Wechsel von der Anwartschaftsdeckung zum Umlageverfahren war zunächst ein unfreiwilliger und wurde mehr stillschweigend denn explizit vollzogen - im Zuge der großen Inflation in den Jahren zwischen 1921 und 1923 (Geyer 1987: 83-108). Die weiterhin vorherrschende Überzeugung, daß das Verfahren der Kapital- oder das der Anwartschaftsdeckung das eindeutig seriösere Finanzierungsverfahren repräsentierte, wurde durch den Umstand herausgefordert, $\mathrm{da} B$ der Staat zwar möglicherweise ein Garant der „nie unterbrochenen Folge versicherter Generationen" (s.o.) war, er aber nicht den Wert des Geldes und damit der angesammelten Kapitalien der Rentenversicherung garantieren konnte (bzw. wollte). Der Nationalstaat wurde zwar zunehmend zum Bezugsrahmen für die Sicherung sozialer Risiken, mit der Aufgabe des internationalen Goldstandardregimes 1914 war der Staat jedoch zugleich zum Garanten des Geldwerts geworden. Bekanntlich hat in Deutschland der Staat in der Erfüllung dieser Aufgabe vollständig versagt.

Der neue Staat, für den im Weltkrieg gekämpft und gestorben werden sollte und wurde, war ein Staat, der kein hinreichendes eigenes Steuereinkommen zur Finanzierung dieses Krieges besaß (vgl. zum Folgenden insbesondere Feldman 1993, Kap. 1). Der Reichshaushalt mußte im wesentlichen aus den Matrikularbeiträgen der Länder finanziert werden, und zur Deckung der enormen zusätzlichen Kosten des Krieges mußte das Reich ,Anleihen' bei seiner Bevölkerung aufnehmen. Reichsanleihen hatten den gesetzlichen Status einer ,mündelsicheren' Anlageform (nach $\S \S 1807-$ 1808 BGB). Gesetzlich waren die Arbeiter- und Angestelltenversicherung ohnehin dazu verpflichtet, mindestens ein Viertel ihrer Kapitalien in solchen mündelsicheren Staatspapieren anzulegen (siehe $\$ 26$ Abs. 1 Satz 1 RVO). ${ }^{9}$ Doch beide Ver-

\footnotetext{
9 Vgl. §226 Angestelltenversicherungsgesetz vom 20. Dezember 1911 (RGBl. I, 989). Nach der Inflation, in der RVO vom 15. Dezember 1924, hieß es dann höchstens $25 \%$ (siehe $\& 27$ f RVO). Diese Bestimmung wurde im Zuge der finanziellen Vorbereitungen für den nächsten Krieg durch die Verordnung über die Anlegung des Vermö-
}

sicherungen überschritten diesen Pflichtanteil im I. Weltkrieg deutlich. An den insgesamt neun Kriegsanleihen zeichneten die Invaliden- und Angestelltenversicherung - ins Verhältnis gesetzt zum Gesamtvolumen aller Kriegsanleihen - zwar keinen besonders gewichtigen Anteil. Gemessen in Prozent ihrer gesamten Kapitalien war ihre Beteiligung jedoch beträchtlich.

Den im Verlauf des Krieges wachsenden Zweifeln an der zukünftigen Einlösbarkeit jener enormen staatlichen Zahlungsversprechen, die die Kriegsanleihen darstellten, wurde von offizieller Seite mit einem Argument begegnet, das im Kontext der früheren Diskussion um die Finanzierungsverfahren in der Rentenversicherung noch ganz abseitig erschienen wäre (s. Feldman 1993: 25-51): Zum einen meinte man, alle Zweifel mit dem Hinweis auf den bevorstehenden ,Siegfrieden ' entkräften zu können, durch den man die Kosten des Krieges auf die Verlierernationen würde abwälzen können. Zum anderen gewann ein neues Argument an Überzeugungskraft, das die Nation als Summe aller ,produktiven und Vermögen schaffenden Fähigkeiten des deutschen Volkes" (Feldman 1993: 47; meine Übersetzung) definierte. Diese Summe fungierte quasi als Sicherheit für die Kriegsanleihen und schien durch die Anleihen lediglich hypothekarisch belastet.

Wie man weiß, waren es die Siegermächte, die ihre Kriegsführungskosten auf Deutschland abzuwälzen suchten, und die Reichsanleihen erwiesen sich als alles andere als ,mündelsicher'. Die Invalidenund Angestelltenversicherung verloren in der groBen Inflation nahezu ihr gesamtes Vermögen, d.h. die durch die Beiträge der Vergangenheit erworbenen Anwartschaften waren nahezu vollständig ohne Deckung (siehe Abbildung 1). Das bürgerliche Projekt einer verantwortungsvollen Planung für die Zukunft durch ,Hortung von Kapitalien schien ad absurdum geführt. Zur Hochzeit der Inflation lautete die Klage: „Die Vorsorge vergangener Zeit war zwecklos, die Vorsorge für kommende Zeiten ist gegenwärtig ebenso zwecklos, unsere Zeit ist ganz allein auf sich selbst gestellt, kann in der Gegenwart nur für die Gegenwart sorgen" (Günther 1923: 4). Aus einer Institution, deren er-

gens der Rentenversicherung vom 14. April 1938 (RGBl. I, 399) auf mindestens $50 \%$ erhöht. Bereits das Gesetz zur Erhaltung der Leistungsfähigkeit der Invaliden-, Angestellten- und der knappschafilichen Versicherung vom 7. Dezember 1933 hatte aus dem „höchstens $25 \%$ “ wieder das vor 1924 geltende „mindestens $25 \%$ “ gemacht $(\$ 22$; vgl. Dobbernack 1934: 80-81). 


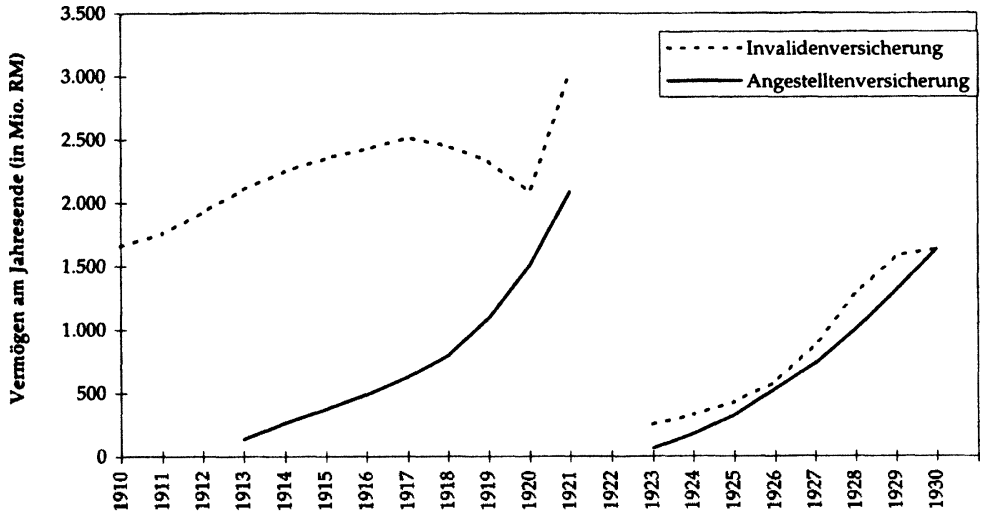

Jahr
Abb. 1 Das Vermögen der Invaliden- und Angestelltenversicherung 1910-1930. (Quelle: Mörschel (1990). ster Zweck die Sicherung gegen zukünftige Risiken war, machte die galoppierende Inflation in der Wahrnehmung der Versicherten durch die rasche und nahezu vollständige Entwertung ihrer Beiträge eine Institution, die lediglich in der Gegenwart „für die zwangsweise Konfiskation von [...] Löhnen" sorgte (zitiert nach Feldman 1993: 559; meine Übersetzung).

Bei der notwendigen Neuordnung der Sozialversicherung im Zuge der Stabilisierung $1924 \mathrm{kam}$ jedoch kaum jemand auf die Idee, daß die durch langjährige Beitragszahlung erworbenen, aber nun durch keinerlei Kapitalreserven mehr gedeckten Anspruiche von Rentnern und Versicherten aus der Summe der ,produktiven und Vermögen schaffenden Fähigkeiten des deutschen Volkes' gedeckt werden könnten. Es war zwar allen Beteiligten vollkommen klar, daß genau dies - die Finanzierung der gegenwärtigen Renten aus dem gegenwärtigen Beitragsaufkommen, also das Umlageverfahren - in der Inflation und der Zeit der Stabilisierung vorherrschende Praxis war (zudem teilweise kombiniert mit der Preisindexierung der Beiträge und der hierdurch ermöglichten ,Dynamisierung' von sogenannten Teuerungszulagen, den alsbald eigentlich wichtigen Bestandteilen der Rentenansprüche; vgl. Geyer 1987). Dennoch schien die Überzeugung von der Überlegenheit der Anwartschaftsdeckung ungebrochen. ,In der Gegenwart nur für die Gegenwart [zu] sorgen', war eine Praxis mit schlechtem Gewissen, geboren aus der Not, und lediglich negativ begründet mit der Notwendigkeit, „das Anwartschaftsdeckungsverfahren auf bessere Zeiten zu vertagen“ (Geyer 1987: 153), hätten doch die Beiträge unerträglich hoch gesetzt werden müssen, wenn man dem Prin- zip der vollständigen Kapitalisierung tatsächlich hätte folgen wollen. Zur Legitimierung verwies man auf die Not der Zeit: Der „Gesetzgeber [hatte] aus dem durch die Inflation geschaffenen Trümmerhaufen ein Notgebäude errichtet, dessen Festigung und Ausbau einer späteren Zeit vorbehalten bleiben sollte“ (Dobbernack 1934: 7).

Wichtig ist nun die Frage, warum diese massivste aller denkbaren Erschütterungen der Lehre von der Überlegenheit der Kapital- oder Anwartschaftsdeckung, die Hyperinflation, offensichtlich als Erfahrung nicht einschneidend genug war, um zur grundsätzlichen Revidierung der herrschenden Lehre zu führen. Wenn die große Inflation noch 1957 bei der Adenauerschen Rentenreform als mächtige Hintergrunderfahrung die Einführung der dynamischen Rente und des (partiellen) Umlageverfahrens motiviert haben soll, so stellt sich die Frage, warum die offensichtliche Absurdität, erst in Zeiten mangelnder Geldwertstabilität und dann in der schwierigen wirtschaftlichen Erholungsphase am Kapitaldeckungsverfahren festzuhalten, nicht schon unmittelbar nach $1923 / 24$ zu einem Umdenken und Umlenken in der Rentenpolitik geführt hatte. Mangel an Verständnis für wirtschaftliche Zusammenhänge scheidet als Erklärungskandidat weitgehend aus, denn schon 1925 konnte man die Einsicht gewonnen haben, daß „gegenüber dem auf privatwirtschaftlichen Gedankengängen aufgebauten Kapitaldeckungsverfahren das Umlageverfahren gesiegt habe, indem erkannt wurde, daß die Quelle der Leistungen der Sozialversicherung das Sozialprodukt ist" (zitiert nach Geyer 1987: 155).

Die Rückkehr zur Kapitaldeckung war Beweis dafür, daß die Inflation in der zeitgenössischen Wahr- 
nehmung nicht unbedingt die Finanzierung der Sozialversicherung als problematisch hatte erscheinen lassen, sondern in erster Linie als Problem der Geldpolitik interpretiert wurde. Wenn durch die Stabilisierung die Inflation besiegt, der Geldwert gesichert schien und wenn nun insbesondere die Autonomstellung der Reichsbank inflationsverhindernd wirken sollte, so standen der Rückkehr zum ,einzig seriösen' Finanzierungsmodus, der Kapitaldeckung, nur noch der Umstand im Wege, daß die hierfür notwendigen sehr hohen Beitragssätze der schwachen Konjunktur schwer schaden würden. Zugleich zeigte die allmähliche Rückkehr zur Kapitaldeckung, daß es jetzt auch entscheidend war, welche Konsequenzen das Votum für Umlage oder Kapitaldekkung für die Verteilung von politischer Macht und Sektorregulierungskompetenz besaß. Tatsächlich wird man erneut in der vorherrschenden Wahrnehmung der Rolle des Staates einen wichtigen Erklärungsfaktor für die Wiedereinsetzung der Anwartschaftsdeckung in der GRV finden können, nachdem die Inflation kurz zuvor alle Behauptungen von der höheren Sicherheit dieses Finanzierungsverfahrens so offensichtlich Lügen gestraft hatte. Der Staat der Weimarer Zeit bildete in den Augen vieler keine staatliche Einheit. Mit dem Ende der Monarchie war diese Einheit für viele unwiederbringlich verloren gegangen. An ihre Stelle war der Weimarer Parteienstaat getreten, in dem sich - wie es bei Carl Schmitt heißt - die Parteien "der staatlichen Willensbildung bemächtigen, ohne aufzuhören, nur soziale (nicht-staatliche) Gebilde zu sein" (Schmitt 1985: 71). Wie sollten diese untereinander zerstrittenen und lediglich flüchtige Koalitionen bildenden Parteien zur langfristigen Selbstbindung fähig sein, langfristige Versprechen honorieren können? In dieser Hinsicht kann die angestrebte erneute Kapitalisierung der Rentenanwartschaften als Entsprechung zu jener nach 1923/24 sprunghaft erweiterten Autonomie von Reichsbank und Reichsverfassungsgericht gegenüber der politischen Sphäre angesehen werden. Eine Autonomie, die ebenfalls mit der Schwäche und Volatilität parteipolitischer Entscheidungsprozesse und der daraus resultierenden geringen Vertrauenswürdigkeit politischer Langfristversprechen begründet wurde. Eine sozialpolitische ,Glaubwürdigkeitslücke' bestand dann nur noch insofern, als das Parlament weiterhin über das Beitrags- und Leistungsrecht der Sozialversicherung bestimmen konnte - und genau hieraus speisten sich die Konflikte der folgenden Jahre.

Tatsächlich bezog das Kapitaldeckungsverfahren seine Legitimation jetzt im wesentlichen aus der neuen Frontstellung zwischen den politischen Par- teien einerseits und der zuständigen Fachbürokratie des Reichsarbeitsministeriums andererseits, zwischen ,ungezügeltem Parlamentarismus“ (Geyer 1991: 415) und der vermeintlich ausschließlich dem Gemeinwohl verpflichteten Beamtenschaft. Die Semantik von verantwortlich versus unverantwortlich, von langfristig versus kurzsichtig, von maßvoller Selbstbeschränkung versus selbstvergessenem Drang nach unmittelbarer Bedürfnisbefriedigung bezog sich jetzt nicht mehr auf die Gegenüberstellung von bürgerlich-verantwortungsvoller Vorsorge für die Zukunft und proletarischem ,Sorglos-inden-Tag-hinein-Leben', sondern auf politische Akteure, deren Handeln von unterschiedlichen Zeithorizonten geprägt war.

Es war die Ministerialbürokratie, die sich wegen der Erfahrung mehrfacher sozialpolitischer Abstimmungsniederlagen im Reichstag zunehmend auf die Seite der Wohlfahrtsstaatskritiker stellte und am Parlament vorbei, z.T. sogar gegen das Parlament, zunehmend mit Hilfe von Notverordnungen, eine strikte Sanierungspolitik zu verfolgen suchte (vgl. Geyer 1991: 415-418). Für die Rentenpolitik bedeutete dies, erneut auf die Anwartschaftsdeckung zu drängen. Als hätte es die Inflation nie gegeben, hieß es nun wieder: „Es ist falsch, Versicherungspolitik nur auf die Zeit einer kurzen Umlageperiode zu machen und die Sorgen der $\mathrm{Zu}$ kunft späteren Generationen zu überlassen; die Versicherung muß eine sichere Dauereinrichtung sein" (Dobbernack 1933: 260-261). Die Ministerialbürokraten hofften auf die in der Anwartschaftsdeckung besonders verkörperte disziplinierende Wirkung des Grundsatzes ,keine Leistungserhöhung ohne die entsprechende Deckung..10 Doch rief die Obszönität relativ hoher Kapitalreserven in der Sozialversicherung in Zeiten einer harten Austeritätspolitik zum Ende der 20er und Anfang der 30er Jahre die Kritik an der ,Thesaurierungspolitik' des Reichs hervor - eine Kritik, die sich bald mit der radikaleren Wohlfahrtsstaatskritik der Nationalsozialisten und Kommunisten vermischte, in deren Lesart die Kapitalien der Sozialversicherung allein der Versorgung einer (angeblich überwiegend sozialdemokratischen und/ oder jüdischen) Funktionärsschicht dienten.

Wenn die Arbeitgeber angesicht der guten Sozialversicherungsfinanzen auf Beitragssenkungen

${ }^{10}$ Konsequent angewandt, erfordert die Anwartschaftsdeckung, bei jeder Leistungsverbesserung für die aktuelle Gemeinschaft der Beitragszahlenden und Rentenempfänger auch die bereits angesammelten Kapitalien entsprechend aufzustocken. 
drängten, die Gewerkschaften Leistungsverbesserungen forderten und die Parteien sich in der Sozialpolitik immer wieder zu sachbezogenen ProWohlfahrtsstaats-Koalitionen formen konnten, während die Ministerialbürokratie vergeblich plausibel zu machen suchte, daß sich selbst bei dem hohen Vermögen der Rentenversicherung eine ,rechnerische Deckungslücke‘ von 19 Milliarden Reichsmark ergebe (Dobbernack 1934: 22), so schien das Finanzierungsverfahren allein nicht in der Lage, die angesammelten Kapitalien der Sozialversicherung vor der Verfügungsgewalt ,kurzsichtiger Parteipolitik' zu schützen. Bezeichnenderweise erfolgte die Rückkehr zur Anwartschaftsdeckung in der Rentenversicherung schließlich durch eines der ersten sozialpolitischen Gesetze des ,neuen Regimes', durch das Gesetz zur Erhaltung der Leistungsfähigkeit der Invaliden-, Angestellten- und der knappschaftlichen Versicherung vom 7. Dezember 1933 (RGBl. I., 1039). Die bürokratisch über Notverordnungen durchgesetzte Sanierungspolitik der „konservativen Stabilisierung" (Geyer 1991) fügte sich somit relativ nahtlos in die nationalsozialistische Sozialpolitik ein, die das Muster der ,Sanierung' bis weit in die zweite Hälfte der 30er Jahre fortsetzte, auch dann noch, als die wirtschaftlichen Bedingungen sich schon grundlegend gewandelt hatten. Der Staat schien zwar unter den Nationalsozialisten seine verlorene innere Einheit zurückerhalten zu haben, doch - wie sich bald erweisen sollte - war es nicht das vordringliche Interesse des neuen Staates, durch glaubwürdige Selbstbindung die in der Vergangenheit erworbenen Rentenanwartschaften $\mathrm{zu}$ sichern. Sein Interesse lag anderswo. Die Kapitalien der Sozialversicherung wurden im Dritten Reich erneut für den Zweck der Kriegsfinanzierung benutzt. ${ }^{11}$ Doch Geschichte wiederholte sich nicht einfach nur.

\section{1935-1969: Nationale Zeit}

Im Unterschied zur Zeit des Ersten Weltkriegs und der Großen Inflation wurden die Gelder der Sozialversicherung unter den Nationalsozialisten nicht heimlich zweckentfremdet und später ,kalt (über die Geldentwertung) enteignet. Vielmehr besaß die Praxis der Nationalsozialisten eine

$1147 \%$ des Vermögens der Invalidenversicherung (1938) und sogar $75 \%$ des Vermögens der Angestelltenversicherung (1943) waren in Form von Reichsanleihen angelegt, d. h. sie dienten vornehmlich der Kriegsfinanzierung (vgl. Mörschel 1990: 635-636, 644).
Theorie - für wie krude oder zynisch man diese auch immer halten mag. Die Nutzung der Sozialversicherungsgelder zur Finanzierung der Kriegsvorbereitungen schien in ihrem Rahmen so legitim, daß sie recht offen thematisiert werden konnte (vgl. Scheur 1967: 164-166; Teppe 1977: 236237; Bühler 1940; AWI 1939).

Theodor Bühler, wissenschaftlicher Generalreferent des Arbeitswissenschaftliche Instituts (AWI) der Deutschen Arbeitsfront, beschrieb als Ziel der neuen, nationalsozialistischen Sozialpolitik, daß sie nicht mehr „defensiv“ ,Mißstände im Gebiet des Verteilungsprozesses mit Mitteln der Gesetzgebung und Verwaltung zu bekämpfen versuchte (Adolph Wagner), sondern daß ihr Wesen in der „Freilegung der nationalen Dynamik“ liege (Bühler 1940: 7). „Ihr letztes Ziel liegt nicht in der Gegenwart, sondern in dem Schicksal der kommenden Generationen. [...] Die Ewigkeit des völkischen Bestands ist für sie kein politisches Schlagwort, sondern der Marschkompaß ihres Handelns" (Bühler 1940: 7). Auch Rudolf Crämer, Referent für Sozialgeschichte im AWI, bezog seine ,moderne' Definition von Sozialpolitik auf die Zukunft „des nationalen Lebenskampfes“ (Crämer 1941: 620). Im Kern seiner umfangreichen, recht geschwollen formulierten Definition stand, „daß Leben und Wohlfahrt, Rechtsschutz, Leistung und Führerschaft im artgebundenen Gemeingeist des Volkstums für die geschichtliche Wirklichkeit von Zeit und Zukunft gesichert werden" (Crämer 1941: 621). Trotz des offensichtlich propagandistischen, z.T. schwammig ideologischen Gehalts dieser Sätze kommt in ihnen doch prägnant eine Konzeption von Zeit zum Vorschein, die Charles Maier „fascist time“ genannt hat (Maier 1987: 161-164): ,[F]ascism was the triumph of the permanent [...] $[\mathrm{T}]$ he party and regime were to be eternal $[\ldots] .^{12}$ On the individual level aging and mortality were to be transcended by climactic sacrificial intensity. On the collective level institutional decay was simply negated“ (Maier 1987: 162-163).

Diese Umdefinition von Zeit beruhte nicht einfach nur auf dem Leugnen von Interessenkonflikten in der Allokation und Nutzung von Zeit durch den Bezug auf das Ziel der Einheit und Ewigkeit des völkischen Bestands, dem alles unterzuordnen war. ${ }^{13}$ Sozialpolitik bekam nun auch einen ande-

12 Man müßte hier ergänzen: Weniger der Staat oder das Regime, sondern das Volk war ewig!

13 „Sozialpolitik im nationalsozialistischen Gesamtziel [hat] den Sinn [...], die Lebensansprüche der Einzelnen dem Gemeinwohl unterzuordnen" (Crämer 1941: 619). 
ren, investiven Stellenwert, und dies beinhaltete modernere Elemente, als der Rekurs aufs, Völkische' zunächst vermuten läßt. Sozialpolitik war nicht etwas die kapitalistische Wirtschaft nur Komplementierendes, Korrigierendes - dabei eine ,soziale Belastung' der Wirtschaft -, sondern integraler Bestandteil und Stimulus der Produktion selbst. Zwar war auch bereits in den sozialpolitischen Debatten der Weimarer Zeit immer wieder auf den ,wirtschaftlichen Wert der Sozialpolitik، (Götz Briefs) verwiesen worden, doch diese Argumentation wurde seitens der Deutschen Arbeitsfront nicht mehr defensiv, sondern ausgesprochen offensiv vorgetragen (vgl. Geyer 1989).

Die grandiosen sozialpolitischen Reformplanungen der Deutschen Arbeitsfront trugen dabei immer den Zusatz: ,nach dem Kriege' (AWI 1940/41: 24-64). Der Krieg selbst erschien als massive Investition in die Zukunft des Volkes zum Zwecke der Mehrung seines Reichtums. „Dabei kann es durchaus Sozialpolitik sein", schrieb Theodor Bühler, „für eine bestimmte Zeit Opfer und Verzicht zu fordern, wenn mit diesen Opfern und Verzichten Grundlagen für eine bessere Entwicklung der Nation erreicht werden können" (Bühler 1940: 17). Der Gratifikationsaufschub war nun der eines ganzen Volkes, und die Nutzung der sich durch die anhaltend restriktive Beitrags- und Leistungspolitik auftürmenden Sozialversicherungskapitalien zur Kriegsfinanzierung war aus dieser Perspektive durchaus folgerichtig. Sie schien sogar aus sozialpolitischen Gründen einer ausschließlichen Steuerfinanzierung des Krieges vorgezogen werden zu müssen. Verwendet der Staat - so hieß es in einer diesbezüglichen Denkschrift des AWI (,Kriegsfinanzierung über die Altersversorgung?"; AWI 1939) - „Sozialversicherungsbeiträge für allgemeine Staatszwecke, so legt er einen Teil seiner zukünftigen Einnahmen für Zwecke der Altersversorgung fest" (AWI 1939: 3). Angesichts der überproportional hohen Belastungen und Entbehrungen der unteren Einkommensschichten in Kriegszeiten schien mit der Finanzierung des Krieges durch angehobene Sozialversicherungsbeiträge ein Weg gegeben - ,wenn schon keine ,,absolute Gerechtigkeit in der Verteilung der Gegenwartslasten zu erreichen" ist - ,wenigstens die Hoffnungen auf die Zukunft, die ja der Krieg begründen soll, so zu verteilen, daß damit ein gewisser Ausgleich für die Härten der Gegenwart geschaffen wird. Nicht, daß man etwa versuchen sollte, durch billige und problematische Versprechungen die notwendigen Einschränkungen mundgerechter zu machen. Wohl aber müßte es möglich sein, schon jetzt das Kriegsfinanzierungssystem so aufzubauen, daß die etwa eintretenden Verbesserungen der völkischen Lebenslage zwangsläufig dann in erster Linie den Kreisen zufallen müssen, die jetzt die härtesten Opfer auf sich nehmen" (AWI 1939: 4; meine Hervorhebung). Die Finanzierung des Krieges durch die Gelder der Altersversicherung war damit als sozialpolitische "Vorgabe für die $\mathrm{Zu}$ kunft" gedacht (AWI 1939: 6), entsprach es doch „durchaus dem Sinn eines sozialistischen Krieges, den endgültigen Erfolg von vornherein bereits in diese Richtung festzulegen" (AWI 1939: 9).

Damit änderte sich auch die vorherrschende Vorstellung von der angemessenen Finanzierungsweise der ,Altersversorgung, wie die Rentenversicherung nun bezeichnenderweise genannt wurde. Wenn die Referenzgröße die Volksgemeinschaft war (wobei die Ausgliederung aller, volksfremden oder gemeinschaftsschädlichen Elemente' aus ihr so selbstverständlich unterstellt war, daß eine besondere Erwähnung überflüssig schien), so war klar, da 3 ,die Alten und Arbeitsunfähigen [...] in jedem Jahr von der jeweils schaffenden Generation erhalten werden müssen“. Hieraus folgte: „Es bedeutet also im Prinzip gar keine Entlastung der Zukunft, wenn die Rentenversicherungsträger sich jetzt Kapitalreserven ansammeln, denn auch in Zukunft können die zur Rentenzahlung erforderlichen Mittel [...] nur aus dem jeweiligen Volkseinkommen stammen. [...] Insofern [...] kommt auch das in der Rentenversicherung angewandte Anwartschaftsdeckungs-Verfahren in güterwirtschaftlicher Hinsicht nur auf ein Umlageverfahren hinaus" (AWI 1940/41: 91-92). Hier entwickelte sich also erstmals eine kraftvolle positive Begründung für das Umlageverfahren.

Dabei werden diese Formulierungen vielen vertraut sein. In der Tat stimmen die im Umkreis des Arbeitswissenschaftlichen Instituts der DAF entwickelten Argumente für das Umlageverfahren z.T. bis in die Wortwahl mit jener sogenannten ,Mackenroth-These überein, mit der Gerhard Mackenroth zwölf Jahre später vor dem Verein für Socialpolitik für so viel Aufsehen sorgen sollte (Mackenroth 1952; vgl. Schmähl 1981; Schewe 1996). Wenn Mackenroth Anfang der Fünfziger ausführte: „Nun gilt der einfache und klare Satz, daß aller Sozialaufwand immer aus dem Volkseinkommen der laufenden Periode gedeckt werden muß", so fällt es in der Tat schwer, gravierende Unterschiede $\mathrm{zu}$ Aussagen $\mathrm{zu}$ identifizieren wie etwa derjenigen Theodor Bühlers von 1940: „Die Versorgung der alten und arbeitsunfähigen Volksgenossen läuft volkswirtschaftlich auf eine ganz 
klare und einfache Formel hinaus: Alles, was die Alten und Arbeitsunfähigen verbrauchen, muß aus dem laufenden Produktionsertrag der Schaffenden abgezweigt werden“ (Bühler 1940: 151; vgl. Schmähl 1981: 162-163). Es scheint also einer jener für die junge Bundesrepublik so wichtigen Nachkriegsmythen zu sein, erst mit der Rentenreform von 1957 sei das Umlageverfahren „durch Kreislauftheorien nationalökonomisch fundiert und durch die Idee des Generationenvertrags normativ abgesichert" worden (Hockerts 1983: 308). Zumindest war die wesentliche argumentative Vorarbeit für das Umlageverfahren bereits von der DAF geleistet worden, ${ }^{14}$ die hierbei eine Argumentation entwickelte, die zeitgleich (1942) auch im Beveridge-Plan ausformuliert wurde: „Der Staat, welcher die Macht hat, aufeinanderfolgende Generationen von Bürgern zur Versicherung zu zwingen und Steuern aufzuerlegen, ist von der Notwendigkeit befreit, Reserven für statistische

14 In zusätzlicher Hinsicht muß man in der Rentenversicherung den wichtigen Einfluß nationalsozialistischer Sozialpolitik auf die Sozialpolitik der frühen Bundesrepublik konstatieren. Erstens: Der staatliche Zuschuß zur Rentenversicherung wurde unter den Nazis erstmals als globaler Zuschuß und nicht mehr als staatlicher Anteil zur einzelnen Rente gezahlt (zugleich wurde der Reichszuschuß erheblich angehoben; vgl. Mörschel 1978). Zweitens: Erstmals wurde die letztendliche Garantie von rentenrechtlichen Ansprüchen nicht mehr durch die Länder oder Kommunalverbände, sondern per ,Reichsgarantie gesichert ( $\$ 1384$ Abs. 2 RVO laut Gesetz über den Ausbau der Rentenversicherung vom 31. Dezember 1937, RGBI. 1, 1393). Schließlich erfolgte mit der zweiten Lohnabzugsverordnung noch ein weiterer wichtiger Rationalisierungschritt. Die Beiträge zur Invaliden- und Angestelltenversicherung wurden angeglichen. Beide wurden nicht mehr nach Lohnklassen, sondern als durchgängig prozentualer Anteil vom Lohn berechnet und direkt vom Lohn abgezogen. An allen diesen Regelungen zeigt sich m.E. eine gewandelte, gestärkte Stellung des Staates in der Sozialversicherung. Die genannten Regelungen wurden allesamt ins Rentenrecht der Bundesrepublik übernommen. Zuletzt muß noch darauf hingewiesen werden, daß es bereits zu den „Grundsätzen einer nationalsozialistischen Altersversorgung“ gehörte, „daß die Lebenshaltung der Arbeitsveteranen nicht allzu stark von der der arbeitenden Volksgenossen abstechen darf" (AWI 1939: 18). Hinsichtlich der Frage, ob man in dem ,Hoffähigmachen' des Umlageverfahrens einen modernisierenden Impuls nationalsozialistischer Sozialpolitik sieht, wird man darauf hinweisen müssen, daB es Hand in Hand ging mit dem Abbau der Rechtsverbindlichkeit von sozialpolitischen Leistungsanspritichen. Insofern hat tatsächlich erst die Adenauersche Rentenreform die Dynamisierung als Rechtsanspruch verwirklicht.
Zwecke anzusammeln" (zitiert nach Hockerts 1982: 336).

Das Votum für die Umlage und gegen die Kapitaldeckung war im Rahmen des nationalsozialistischen ,Überzeugungssystems* nur folgerichtig. Dort, wo alles dem Volk und der Hebung seiner Leistungsfähigkeit untergeordnet war und folglich auch Sozialpolitik und „Wehrpolitik“" in innigem Zusammenhang standen (Bühler 1940:13), war der volkswirtschaftliche Reichtum immer ein gemeinsames Jetzt-Produkt, dessen Verteilung auf ,Schaffende' und ,Arbeitsveteranen' sich allein nach der persönlichen abgeleisteten (Arbeits-)Pflicht im Dienste der Gemeinschaft und der umgekehrten Anerkennung der Volksgemeinschaft für diese Verdienste bemaß. ${ }^{15}$ Die Zeitperspektive hatte sich sichtbar verschoben: Der Staat fungierte nicht mehr als Garant für die langfristige Stabilität und finanzielle Solidität individueller oder kollektiver Versicherungen (also als, enforcing third party'), sondern der anwachsende Reichtum des Staates selber - der mit dem ,Volk' als identisch vorgestellt wurde - sollte die Aussicht auf die Vernichtung der Zeitlichkeit der Zukunft bieten. Dem lag ein Wechsel in der argumentativen Referenzgröße zugrunde: „Die Einzelperson kann in der modernen Wirtschaft Geld oder Güter nach freiem Belieben sparen [...] Nehmen wir dagegen das Volk als Ganzes, so kann es ausschließlich in Gütern sparen, nicht in Geld“ (Bühler 1940: 152; gesperrt im Original). Wenn man diese Aussage wiederum der angeblich ,klassischen' Formulierung Winfried Schreibers von 1955 gegenüberstellt - „Der einzelne kann Vermögen anhäufen, um es im Alter zu verzehren, die Gesamtheit des Volkes kann es nicht" (zitiert nach Heine 1988: 434) -, zeigt sich auch hier, wie wenig zutreffend die retrospektive Einschätzung ist, bis 1957 habe „durchweg die volkswirtschaftliche Betrachtung" in der Rentenpolitik gefehlt (Schewe 1963: 389; meine Hervorhebung).

Bei dem erneuten Streit um das beste Finanzierungsverfahren für die gesetzliche Rentenversicherung ging es nicht nur um die Einspeisung neuerer ökonomischer Kreislauftheorien in die Betrachtung der Sozial- und Rentenpolitik, obwohl auch das eine Rolle spielte. Die DAF war insbesondere deswegen in der Lage, das statische, „vorkeynesianische“ (Hockerts 1983: 308) Denken der vorwiegend juristisch geschulten Ministe-

15 Die staatliche Altersversorgung firmierte nun unter dem Titel: „Dank der Volksgemeinschaft für ein treues Staatsbürgerleben" (Bühler 1940: 154). 
rialbürokratie des Reichsarbeitsministeriums (RAM) herauszufordern, weil sie über ihr Arbeitswissenschaftliches Institut (AWI), das sich das Berliner Institut für Konjunkturforschung einverleibt hatte und ohnehin extrem ressourcenreich war, die Elite der nicht emigrierten Nationalökonomen zur Mitarbeit gewinnen konnte (vgl. Roth 1993). Doch ökonomische Theorien traten hier immer auch "als politische Interessen" auf (Krohn 1983). Insofern ging es im Streit zwischen der RAM-Fachelite einerseits und den Reformern der Deutschen Arbeitsfront andererseits auch um die Vorherrschaft bei Politikformulierung und Sektorregulierung. Wenn die RAM-Ministerialbürokratie mit der Strategie der ,konservativen Stabilisierung' gegenüber den Weimarer Parteien schließlich den Sieg davongetragen hatte, vor allem durch die „Notverordnungspraxis parlamentsunabhängig und normbestimmend geworden war" (Hockerts 1983: 307; s.o), so mußte sie je länger, desto deutlicher im Kräftemessen mit der DAF zurückstecken, die im Kampf mit dem Reichsarbeitsministerium auch von Fall zu Fall auf die Unterstützung durch andere, der sozialpolitischen Linie des RAM ohnehin feindlich gesonnenen Ressorts rechnen konnte. Bezüglich der Absicht des RAM, durch Beitragssenkungen bei der Arbeitslosenversicherung kompensierende Beitragserhöhungen in der Rentenversicherung zu ermöglichen und damit der Anwartschaftsdeckung aller Rentenansprüche einen Schritt näher zu kommen, mußten sich die RAMBürokraten sagen lassen, daß die Anwartschaftsdeckung ,in einem parlamentarisch-demokratisch regierten Staat angebracht sein [mag], und zwar zum Schutz gegen die sozialversicherungsfeindlichen Mehrheitsbeschlüsse. Im nationalsozialistischen Staat ist ein solches Verfahren nicht erforderlich, weil es nach nationalsozialistischer Staatsauffassung selbstverständlich ist, daß im Fall eines auftretenden Mehrbedarfs für die erforderliche Deckung gesorgt wird. Das kann am einfachsten durch Anpassung des Reichszuschusses an den Mehrbedarf geschehen" (so der Staatssekretär des Reichsfinanzministeriums Reinhard, zitiert nach Scheur 1967: 111-112).

Es ist nicht ohne (bittere) Ironie, daß die von den Nationalsozialisten unablässig beschworene, völkische Schicksalgemeinschaft' durch die Niederlage in dem von ihnen angezettelten Krieg eigentlich erst entstand. Im Kontext von Wiederaufbau und Aufschwung gewann dann die Vorstellung eines alle einbeziehenden nationalen Positiv-Summen-Spiels viel eher Plausibilität als im Kontext eines sozialpolitisch verbrämten imperialistischen Krieges. Gravierende
Verteilungskonflikte wurden in der unmittelbaren Nachkriegszeit zunächst zurückgestellt und waren anschließend im Wirtschaftswunder-Deutschland nicht drängend - weil nun schließlich alle hinzugewannen. Für die frühe Verteilungsproblematik hingegen wurden vornehmlich sozialpolitische Lösungen gesucht. Vor allem der Konflikt zwischen Kapital und Arbeit war abgemildert, da die Gewerkschaften in ihrer Tarifpolitik wegen des extremen Arbeitsplatzmangels Lohnerhöhungen zunächst nicht als primäres Ziel definierten. Dies erhöhte-kompensatorisch - die gewerkschaftliche Mobilisierung für die gesetzliche Gewährleistung sozialpolitischen Fortschritts (Kampf um die Mitbestimmung und Selbstverwaltung 1951-1952, Lohnfortzahlung 1956, Rentenreform 1957, Protest gegen die Leistungskürzungen der Blank-Reform 1959-1961). Dabei schien die Quelle für die so sehnlich erwartete soziale Sicherheit angesichts von Niederlage und Zerstörung in ganz offensichtlicher Weise in nichts anderem als dem Ertrag der ,nationalen Arbeit' zu liegen. Nicht zufällig klang es daher wie eine Paraphrase auf Bühler, wenn Oswald von Nell-Breuning 1955 ausführte: „Um den Lebensstandard der heute erwerbsfähigen Generation zu sichern, ist die Kapital- und Anwartschaftsdeckung überflüssig, ja nutzlos; nötig ist, die Produktivität unserer Wirtschaft zu erhalten oder zu steigern" (zitiert nach Nullmeier und Rüb 1993:376377) ${ }^{16}$ Die schließlich mit der Rentenreform eingeführte dynamische Rente ließ sich folgerichtig als „eine Art Dividende aus dem Kapital des deutschen Volkes, nämlich seiner Volkswirtschaft",interpretieren (Liebing 1966: 72).

${ }^{16}$ Der Nachsatz bei Nell-Breuning - „[B]enötigt wird eine nachwachsende Generation, deren Arbeitsfähigkeit und Arbeitswille allein dafür gut stehen, daß auch in $\mathrm{Zu}$ kunft Güter verfügbar sind" (Oswald von Nell-Breuning 1955, zitiert nach Nullmeier/ Rüb 1992: 377) - ist dann bekanntlich für die Entwicklung des bundesdeutschen Wohlfahrtsstaats nicht prägend geworden. Bevölkerungs- und familienpolitische Uberlegungen hatten lange Zeit keinen Platz in der Rentenpolitik. Ihre Integration in das Leistungs- und Beitragsrecht der Rentenversicherung wurde lange Zeit als ,systemwidrig“ abgelehnt. Die gängige Rechtfertigung lautete, Bevölkerungspolitik sei eine, gesamtgesellschaftliche Aufgabe, die alle angehe' - also keinen. Familienpolitik ressortierte in dem extrem schwachen und kein eigenes verbandliches oder institutionelles Adressatenfeld besitzenden Familienministerium. Sie war somit außerhalb der ,klassischen' Sozial(versicherungs)politik angesiedelt, profitierte deswegen beispielsweise auch nicht von einem ,spill over' der hier herrschenden Strukturen und Ordnungsprinzipien (bspw. ist das Kindergeld einer der wenigen nicht-dynamisierten Sozialtransfers). 
Auch für Mackenroth war die wichtigste Schlußfolgerung aus seiner, Sozialaufwands-These ${ }^{6} \mathbf{z u -}$ nächst die gewesen, daß die Fondsbildung generell, in der gegenwärtigen ökonomischen Lage Deutschlands aber auch „Konsumumschichtungen“ oder die „Beschneidung der Investitionsrate", keine spürbaren Steigerungen des Sozialaufwands erlauben würden (vgl. Mackenroth 1952: 46-47). ,So bleibt also wirklich nur die Steigerung des Sozialprodukt als Quelle einer Steigerung des Sozialaufwandes." Automatisch stellte sich damit an die Sozialpolitik die Forderung, ,daß sie in ihren Maßnahmen nichts enthält, das mit der Produktivität der Wirtschaft und mit der Steigerung des Sozialprodukts in Konflikt gerät“ (Mackenroth 1952: 47). In der Auseinandersetzung mit der grundlegenden Gegenkonzeption des Bundeswirtschaftsministeriums, das in einer Art, Volkskapitalismus ${ }^{6}$ - also durch breit gestreute Anteilscheine am wirtschaftlichen Aufschwung - das wahre sozialpolitische Ethos verwirklicht sah (s. Abelshauser 1996), konnte sich die vom Bundesarbeitsministerium (BMA) favorisierte Lösung im Rahmen der ,klassischen'Sozialversicherung (nun modernisiert durch Lohnanbindung und Umlageverfahren) wohl insbesondere auch wegen dieses engen argumentativen Nexus zu Produktivitätssteigerungen durchsetzen. Zumindest konnten sich beide sozialpolitischen Konzeptionen gleichermaßen mit der populären Aussage eịverstanden erklären, daß ,eine gute Wirtschaftspolitik immer noch die beste Sozialpolitik' sei. Die neue lohnindexierte Rente wurde folgerichtig zunächst ,Produktivitätsrente“ getauft - eine Sprachregelung, die schließlich wegen der hiermit möglicherweise genährten $\mathrm{Be}$ fürchtungen über eine inflationistische Spirale zwischen Produktivität, Löhnen, Renten und Preisen zugunsten der Wortprägung, dynamische Rente aufgegeben wurde.

In letzter Konsequenz hatten die Planungen der DAF ein staatliches Versorgungswerk zum Ziel, über Steuern finanziert, gestützt durch eine ,Staatsgarantie' für alle Staatsbürger bzw. Volksgenossen mit stärker nivellierendem Leistungsrecht. Dieser Weg ist nach dem Krieg in Westdeutschland ganz bewußt nicht weitergegangen worden. Staatliche Lösungen waren nach 1945 im Westen doppelt diskreditiert: ,Weder NS noch DDR' lautete die richtungsweisende Formel in der Aufbauphase der Nachkriegsbundesrepublik (vgl. Manow 1997). Während die kraftvolle wohlfahrtsstaatliche Expansion in den meisten europäischen Ländern nach dem Zweiten Weltkrieg staatlich initiiert und geleitet war, folgte die Bundesrepublik einem eige- nen, eigentümlich gegenzyklischen Entwicklungspfad. In West-Deutschland lag der Akzent nun wieder auf Selbstverwaltung, parafiskalischem Status, eher Staatsferne. Die Renten wurden 1957 stärker nach Einkommen und Beiträgen differenziert: Das Versicherungsprinzip sollte in den Vordergrund treten. Der Staat nahm sich in seiner Bedeutung zurück, er beaufsichtigte nun gleichsam nur den ,Vertrag zwischen den Generationen', der aber als weitestgehend, selbst-tragend' und stabil vorgestellt wurde aufgrund der „Gewißheit der Kontinuität des Volksdaseins“ (W. Schreiber zitiert nach Heine 1988: 432). ${ }^{17}$ Wie bei Zentralbank und Verfassungsgericht knüpfte die Bundesrepublik auch in der Sozialpolitik an die in der Weimarer Republik ausgebildete Tradition einer durch verschiedene Bereichssouveränitäten gebrochenen zentralstaatlichen Souveränität an (vgl. Schmidt 1990). In Übereinstimmung hiermit, wenn auch in gewandelter institutioneller Form, etablierte sich auch in der Rentenversicherung ein neues Gleichgewicht von Staat und Gesellschaft. Die Eigenständigkeit der Rentenversicherung wurde nun nicht mehr in der parafiskalen Fondsbildung, sondern in einer dynamischen, quasi-automatischen Anpassungsformel gesehen. Hiermit glaubte man die entscheidendere Variable der parteipolitischen Verfügungsmacht entzogen zu haben (Schewe 1963). Zudem war das Vermögen der Rentenversicherung nun zum zweitenmal nahezu vollständig verloren gegangen (siehe Abbildung 2).

Zu dem Zeitpunkt schließlich, als die erste Rezession in Nachkriegsdeutschland eine der unausgesprochenen Voraussetzungen für das Funktionieren des generös ausgestatteten, Generationenvertrages $^{6}$ in Frage stellte, schrieb das Dritte Rentenversicherungs-Änderungsgesetz von 1969 das reine Umlageverfahren mit einjährigem Beitragsabschnitt für die Rentenversicherung fest (1957 war ein auf zehn Jahre berechnetes Abschnittdekkungsverfahren, also ein modifiziertes Umlageverfahren, eingeführt worden). Die hierfür angeführte Begründung war nun das genaue Gegenteil jener Befürchtungen, die ,Gegenwart auf Kosten späterer Jahrzehnte zu entlasten', die am Anfang der Rentengesetzgebung in den 80er Jahren des

17 Überspitzt, doch im Kern zutreffend heißt es bei Michael Stolleis, durch das Umlageverfahren sei die Rentenversicherung nun keine „Zwangssparkasse mehr", sondern eine „prekäre 3-Generationen-Solidarität, die sich des Staats als clearing-Stelle zur Erhaltung der Liquidität bedient" (Stolleis 1984: N 34). 
Abb. 2 Das Vermögen der Invaliden- und Angestelltenversicherung 1924-1956.

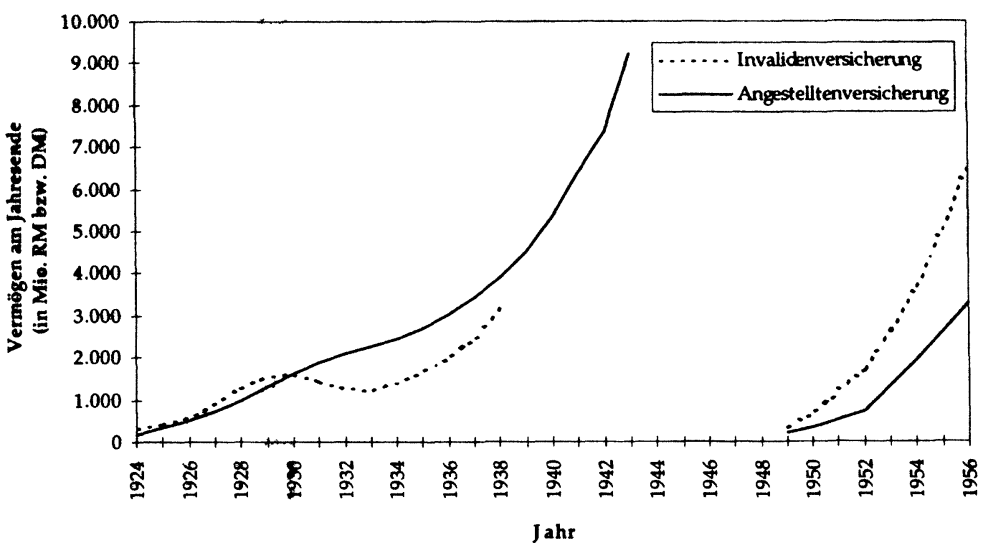

19. Jahrhunderts gestanden hatten. Nun hieß es: „Bei der künftigen Zunahme der Altersbelastung führt jede Beitragsfestsetzung für einen zehnjährigen Deckungszeitraum zu einem unerwünschten Ergebnis. Steigt nämlich die Belastung [...], so muß der durchschnittliche Beitragssatz für zehn Jahre höher liegen als derjenige Beitragssatz, der für das erste Jahr oder die ersten Jahre benötigt würde. Es erscheint aber unzweckmäßig, von Versicherten und Arbeitgebern mehr Mittel zu erheben, als in den nächsten übersehbaren Jahren unbedingt erforderlich sind. [...] Auch ist eine höhere Belastung der Entgelte bei angestiegenem Wohlstand in späteren Jahren leichter zu ertragen" (BR. Drks. 277/66). Hinzu kam, daß die neue keynesianische Heilslehre, die von nun an die ,Vernichtung der Zeitlichkeit der Zukunft' in wirtschaftspolitischer Hinsicht versprach, es als legitim erscheinen ließ, schon einmal in der Gegenwart den dabei in Aussicht stehenden sozialpolitischen Ertrag zu verteilen (vgl. Hockerts 1992).

\section{1969-...: Individuelle Zeit}

Mit dem Dritten Rentenversicherungs-Änderungsgesetz, spätestens aber mit der Rentenreform von 1972 war sicherlich ein Umschlagpunkt erreicht. Unmittelbar auf die allgemeine rentenpolitische ,folie' von 1972 (vgl. Hockerts 1992) folgte die erste Ölkrise. Der Glaube an die Konjunkturbeherrschung durch keynesianisches deficit spending verflüchtigte sich rasch. Die Zeit der sozialpolitischen Kürzungsgesetze begann Mitte der siebziger Jahre mit den ersten Haushaltsgesetzen. Die kurz darauf einsetzenden Bemühungen um den Schutz der Renten vor diesen Kürzungsgesetzen bedienten sich der beiden institutionellen
Rollenmodelle, die in Deutschland für ,nicht-majoritäre' Politik bereitstehen: des Bundesverfassungsgerichts und der Bundesbank. Während einerseits versucht wurde, für sozialrechtliche Leistungsansprüche einen verfassungsrechtlich verbürgten Eigentumsschutz zu konstruieren (vgl. Stober 1982), der die politische Diskretionsgewalt über die doch durch ,eigene" Beitragsleistungen ,erworbenen' Rentenansprüche entscheidend beschränken sollte, hofften andere, mit dem Wechsel zurück zur Kapitaldeckung in Kombination mit einer analog zum Zentralbankrat geschaffenen Institution eines „Rentenzentralrats“ sei die langfristige Sicherung der Renten in den Zeiten der Fiskalkrise zu erreichen (vgl. Nullmeier und Rüb 1993: 385-386).

Damit hat sich die Diskussion um die richtige Finanzierung der Rentenversicherung erneut verschoben. In ihr besteht heute zwischen den Befürwortern wie Gegnern des Umlageverfahrens weitgehende Übereinstimmung im Hinblick auf die Frage, worin denn nun die eigentliche rentenpolitische Problematik bestehe. Heute lautet die vorherrschende Überzeugung auf beiden Seiten: ,the faltering of intertemporal commitment [cannot] be overcome by democratic politics. The state can coerce present time but not future time" (Maier 1987: 169; meine Hervorhebung). Die Kontroverse um Umlage oder Kapitaldeckung wird vorwiegend als Debatte um die richtige Therapie gegen die notorische Willensschwäche der Politik geführt - Kapitalbildung in autonomen, staatsfernen Fonds oder Vermeidung jeglicher Kapitalbildung, also Umlage? Als Befürworter des Status quo und Gegner eines Wechsels zur Kapitaldeckung meint Franz Ruland, immerhin Direktor und stellvertretender Geschäftsführer des Verbandes Deutscher 
Rentenversicherungsträger (VDR), ${ }^{18}$ eine französische Spruchweisheit zitieren zu müssen: Politik, das sei „das Geld der anderen“ (Ruland 1988: 110). Und ganz in diesem Sinne verteidigt auch Bert Rürup, ebenfalls dem engeren Kreis des rentenpolitischen Politiknetzwerks zugehörig und ein Gegner der Kapitaldeckung, das Umlageverfahren gegen seine Kritiker. Er rekurriert auf den Satz Werner Sombarts, daß sich eher ein „Mops einen Vorrat an Würsten anlegt, als daß ein Politiker [...] Geld, welches ,erreichbar' ist, nicht verausgabt" (Rürup 1995: 725). Der Staat, so sieht man, ist heute weit davon entfernt, als ,permanent identische Person' gedacht zu werden. Er ist heute eher ein ,Lump“, dessen Handeln von Politikern bestimmt wird, die in den Zwängen ,des Kurzstreckenlaufs der Legislaturperioden“" (Stolleis 1984: N13) verfangen sind. Zudem hört der Nationalstaat bzw. der nationale Wohlfahrtsstaat angesichts europäischer Integration und zunehmender weltwirtschaftlicher Verflechtung auf, ein unproblematischer Referenzpunkt für ,gesellschaftliche Integration" zu sein (Kaufmann 1997, 1997a). Der Nationalstaat bietet nicht mehr fraglos den ,kollektiven Bezugsrahmen für die Identität' (s.o.). Im Gegenteil: Es zählen nur noch die konkreten staatlichen Leistungsfunktionen, deren Erfüllung so leidenschaftslos mit den Vorteilsversprechen alternativer Governance-Modi verglichen wird, wie zugleich leidenschaftlich die Letztverantwortung der Politik und die Letztzuständigkeit des Staates für alle auf anderem Wege nicht behebbaren sozialen Mißstände eingeklagt werden.

Bezieht man das Geschilderte zurück auf eine Theorie der Institution und des institutionellen Wandels, auf eine Theorie der Synchronisierung von individueller und institutioneller Zeit und der in Institutionen verkörperten Zeitordnung, wird man sagen können, daß die institutionenökonomische Analyse zu kurz greift, wenn sie allein mit den Kategorien rationaler Erwartung und rationalen Vertrauens auszukommen meint. Demgegenüber betont die soziologische Institutionentheorie bzw. der soziologische Institutionalismus zu Recht den normativen Kerngehalt, der in Institutionen verkörpert ist (vgl. DiMaggio und Powell 1991; Lepsius 1990; Offe 1996). Vorstellungen zeitlicher Ordnung, die immer zugleich auch Vorstellungen gesellschaftlicher Ordnung sind, prägen entscheidend die Wahrnehmung der Akteure über die

${ }^{18}$ Der VDR ist zwar ein eingetragener Verein, ubernimmt aber gesetzlich definierte Funktionen (siehe etwa $\S 146$ SGB VI). langfristige Tragfähigkeit und Tauschgerechtigkeit institutionell vermittelter Kontrakte zwischen den Personen, die in verschiedenen Rollen als Mitglieder, Klienten, Beitragszahler oder Leistungsempfänger an eine bestimmte Institution oder einen korporativen Akteur, an eine ,moralische Person“ also, gebunden sind. Im Falle der sozialen Rentenversicherung kam diesen Vorstellungen zeitlicher und gesellschaftlicher Ordnung über die Zeit hinweg unterschiedliche Plausibilität $\mathrm{zu}$, weil die der Frage nach einer praktikablen und gerechten Form der Risikoteilung zugrundeliegenden gesellschaftlichen Zurechnungsgrößen wechselten.

Doch die Gegenüberstellung von nutzenkalkulierendem Handeln einerseits und wechselnden Normenordnungen andererseits, in die dieses nutzenkalkulierende Handeln sich jeweils eingebettet findet, ist nicht das einzige Ergebnis der obigen $\mathrm{Re}$ konstruktion der Debatte um Kapitaldeckung und Umlage. Aus dem Geschilderten wurde auch deutlich, daß die Uminterpretation vorherrschender Normenbestände, ihre Herausforderung durch konkurrierende Entwürfe oder das subversive Unterlaufen bestehender Ordnungen immer interessengeleitete Unternehmungen sind. Sie werden unternommen von Akteuren, die Einfluß und Macht suchen, ihre Domänen erweitern oder abschotten wollen: ihre ,berechenbaren Dauerbedürfnisse“ (Max Weber) zu befriedigen suchen, also ihre Interessen verfolgen. Wenn die Ministerialbürokratie des Reichsarbeitsministeriums eine sozialpolitische Austeritätspolitik gegen die Weimarer Parteien durchzusetzen suchte, die Deutsche Arbeitsfront die Vormachtstellung des Reichsarbeitsministeriums durch Allianzbildung mit anderen Ressorts und mit Unterstützung durch akademische Expertise brechen wollte oder das Bundesarbeitsministerium schließlich den Einfluß der Parteien und anderer Ressorts durch die Rentenindexierung zu beschränken versuchte, mußte dies jedoch immer mit Rekurs auf plausible, erfahrungsgesättigte oder mit grundsätzlichen $\mathrm{Ge}$ rechtigkeits- und gesellschaftlichen Ordnungsvorstellungen kompatible Deutungen, wie die Welt ist' oder ,wie die Welt sein soll' geschehen. Normen- und Nutzenorientierung stehen somit in einem komplizierten Verhältnis zueinander. Institutionen erlauben und ermöglichen Zielverfolgung innerhalb eines bestimmten Normensets. Akteure, für die eine Veränderung der bestehenden Institutionenordnung von instrumentellem Nutzen wäre, müssen ihre Veränderungsinteressen jedoch mit Bezug auf ein alternatives Set von generellen Normen oder eine alternative ,Funktionenlehre' be- 
gründen, damit institutionelle Reformen mehrheitsfähig werden. Dieser Bezug auf andere Normen oder eine andere Funktionenlehre muß dabei anschlußfähig sein an grundsätzliche Erfahrungen oder weitverbreitete, plausible Gerechtigkeitsstandards.

Die Debatte um die Kapitaldeckung oder das Umlageverfahren bot und bietet immer wieder Gelegenheit für Referenzen aufs Grundsätzliche, weil den beiden Positionen in dieser Debatte selbst grundlegende, ,archetypische' soziale Betrachtungsstandpunkte entsprechen: der individuelle Standpunkt (hier wird die Semantik von Sparen und Ertrag verwendet) und der kollektive (hier wird die Verteilung eines gesellschaftlichen $\mathrm{Ge}$ samtertrags diskutiert). Da sich somit in der Struktur der Debatte um Kapitaldeckung oder Umlageverfahren die beiden grundsätzlichen Pole widerspiegeln, die in einer langfristig stabilen Sicherung des Altersrisikos miteinander in Einklang gebracht werden müßten: Individuum und gesellschaftliches Kollektiv, kann dieser Debatte - ganz anders als , der Rente - eine recht sichere Zukunft vorausgesagt werden.

\section{Literatur}

Abelshauser, W., 1996: Erhard oder Bismarck? Die Richtungsentscheidung der deutschen Sozialpolitik am Beispiel der Reform der Sozialversicherung in den fünfziger Jahren. Geschichte und Gesellschaft 22: 376-392.

AWI [Arbeitswissenschaftliches Institut der Deutschen Arbeitsfront], 1939: Kriegsfinanzierung über die Altersversorgung? Berlin, November 1939. Faksimile in: Hamburger Stiftung für Sozialgeschichte des 20. Jahrhunderts (Hrsg.), Sozialstrategien der Deutschen Arbeitsfront. Bearbeitet und eingeleitet von Karl Heinz Roth, Karsten Linne und Michael Hipp. München: Saur. Teil B (Periodika, Denkschriften, und Gutachten ...), Micro-Fiche Ausgabe, Fiche 130, B1. 656-688 (im Bundesarchiv Koblenz R 2 Nr. 24248).

AWI [Arbeitswissenschaftliches Institut der Deutschen Arbeitsfront] (Hrsg.), 1940/41: Jahrbuch 1940/41, I. Band. Berlin: Arbeitswissenschaftlicher Verlag. Darin insbesondere: „Überblick über die für ein Versorgungswerk möglichen Finanzierungssysteme“, 97-110, und „Die sozialen Aufgaben nach dem Kriege. Versuch eines systematischen Überblicks über ein sozialpolitisches Programm", 24-64.

Bartolini, S., 1993: On Time and Comparative Research. Journal of Theoretical Politics 5(2): 131-167.

Bosse, R./von Woedtke, E., 1891: Das Reichsgesetz betreffend die Invaliditäts- und Altersversicherung vom 22. Juni 1889 erläutert von Dr. R. Bosse und E. von Woedtke. Leipzig: Duncker \& Humblot.
Bühler, T., 1940: Deutsche Sozialwirtschaft. Ein Überblick über die sozialen Aufgaben der Volkswirtschaft. Stuttgart/Berlin: Kohlhammer.

Crämer, R., 1941: Vom geschichtlichen Begriff der Sozialpolitik. S. 582-633 in AWI [Arbeitswissenschaftliches Institut der Deutschen Arbeitsfront] (Hrsg.), Jahrbuch 1940/41, I. Band. Berlin: Arbeitswissenschaftlicher Verlag.

Dasgupta, P., 1988: Trust as a Commodity. S. 49-72 in: Diego Gambetta (Hrsg.), Trust. Making and Breaking Cooperative Relations. Oxford: Basil Blackwell.

Dobbernack, W., 1933: Entwicklung und Stand der Sozialversicherungsfinanzen unter besonderer Berücksichtigung der Rentenversicherungen. Reichsarbeitsblatt IV (15/1933): 254-266.

Dobbernack, W., 1934: Die Rettung der Rentenversicherung. Die finanzielle Neuordnung der Invaliden-, Angestellten- und knappschaftlichen Pensionsversicherung. Stuttgart: Kohlhammer.

Döhler, M./Manow, P., 1997: Strukturbildung von Politikfeldern. Das Beispiel bundesdeutscher Gesundheitspolitik seit den fünfziger Jahren. Opladen: Leske + Budrich.

Elster, J./Slagstad, R. (Hrsg.), 1988: Constitutionalism and Democracy. Cambridge: Cambridge University Press.

Feldman, G.D., 1986: The Fate of the Social Insurance System in the German Inflation, 1914 to 1923 . S. 433-447 in Feldman, G.D./Holtfrerich, C.L./Ritter, G.A./Witt, P.C. (Hrsg.), Anpassung an die Inflation. Berlin/New York: de Gruyter.

Feldman, G.D., 1993: The Great Disorder. Politics, Economics, and Society in the German Inflation, 1914 1924. New York/Oxford: Oxford University Press.

Geyer, M.H., 1987: Die Reichsknappschaft. Versicherungsreformen und Sozialpolitik im Bergbau 19001945. München: Beck.

Geyer, M.H., 1989: Soziale Sicherheit und wirtschaftlicher Fortschritt: Überlegungen zum Verhältnis von Arbeitsideologie und Sozialpolitik im Dritten Reich. Geschichte und Gesellschaft 15: 382-406.

Geyer, M.H., 1991: Soziale Rechte im Sozialstaat: Wiederaufbau, Krise und konservative Stabilisierung der deutschen Rentenversicherung 1924-1937. S.406-434 in Tenfelde, K. (Hrsg.), Arbeiter im 20. Jahrhundert. Stuttgart: Klett-Cotta.

Günther, E., 1923: Die Anpassung der Sozialversicherung an die Geldentwertung und Lohnsteigerung. Jahrbücher für Nationalökonomie und Statistik 121: 1-54.

Hardin, R., 1990: Trusting Persons, Trusting Institutions. S. 185-209 in Zeckhauser, R.J. (Hrsg.), Strategy and Choice. Cambridge, MA: MIT-Press.

Hardin, R., 1992: The Street-Level Epistemology of Trust. Analyse + Kritik 14: 152-176. Auch erschienen in Politics \& Society 21 (1993): 505-529.

Hardin, R., 1996: Institutional Morality. S. 126-153 in Goodin, R.E. (Hrsg.), The Theory of Institutional Design. Cambridge: Cambridge University Press.

Heine, W., 1988: Zur Verläßlichkeit von Versicherungsregelungen bei langen Zeiträumen. Aus juristischer Sicht 
am Beispiel der gesetzlichen Rentenversicherung. S. 431-451 in Rolf, G./Spahn, P.B./Wagner, G. (Hrsg.), Sozialvertrag und Sicherung. Zur ökonomischen Theorie staatlicher Versicherungs- und Umverteilungssysteme. Frankfurt a.M.: Campus.

Hockerts, H.G., 1980: Sozialpolitische Entscheidungen im Nachkriegsdeutschland. Alliierte und deutsche Sozialversicherungspolitik 1945 bis 1957. Stuttgart: KlettCotta.

Hockerts, H.G., 1982: Deutsche Nachkriegssozialpolitik vor dem Hintergrund des Beveridge-Plans. Einige Beobachtungen zur Vorbereitung einer vergleichenden Analyse. S. 325-350 in Mommsen, W.J. in Zusammenarbeit mit W. Mock (Hrsg.), Die Entstehung des Wohlfahrtsstaats in Großbritannien und Deutschland 18501950. Stuttgart: Klett-Cotta.

Hockerts, H.G., 1983: Sicherung im Alter. Kontinuität und Wandel der gesetzlichen Rentenversicherung 1889 - 1979. S. 330-357 in Conze, W./Lepsius, M.R. (Hrsg.), Sozialgeschichte der Bundesrepublik Deutschland. Beiträge zum Kontinuitätsproblem. Stuttgart: KlettCotta.

Hockerts, H.G., 1992: Vom Nutzen und Nachteil parlamentarischer Parteienkonkurrenz. Die Rentenreform 1972 - Ein Lehrstück. S. 903-934 in Bracher, K.D./Mikat, P./Repgen, K./Schumacher, M./Schwarz, H.-P. (Hrsg.), Staat und Parteien. Festschrift für Rudolf Morsey zum 65. Geburtstag. Berlin: Duncker \& Humblot.

Kaufmann, F.-X., 1973: Sicherheit als soziologisches und sozialpolitisches Problem. Stuttgart: Enke.

Kaufmann, F.-X., 1997: Schwindet die integrative Funktion des Sozialstaats? Berliner Journal für Soziologie 1/ 97: 5-19.

Kaufmann, F.-X., 1997a: Herausforderungen des Sozialstaats. Frankfurt a.M.: Suhrkamp.

Köcher, R., 1997: Vor den Reformen. Die Bevölkerung unterschätzt die Tragweite der geplanten Steuer- und Rentenreform. Eine Dokumentation des Beitrags in der FAZ Nr. 12 vom 15. Januar 1997. Institut für Demoskopie, Allensbach.

Kohli, M., 1989: Moralökonomie und »Generationenvertrag «. S. 532-555 in Haller, M./Hoffmann-Nowotny, H.J./Zapf, W. (Hrsg.), Kultur und Gesellschaft. Verhandlungen des 24. Deutschen Soziologentags, des 11. Österreichischen Soziologentags und des 8. Kongresses der Schweizerischen Gesellschaft für Soziologie in Zürich 1988. Frankfurt a.M.: Campus.

Krohn, K.D., 1981: Wirtschaftstheorien als politische Interessen. Die akademische Nationalökonomie 19181933. Frankfurt a.M./New York: Campus.

Lehmbruch, G., 1979: Parteiensystem und Interessenverbände in der Politikentwicklung. Universităt Konstanz. FB Politische Wissenschaft, Diskussionsbeitrag 6/79.

Lepsius, M.R., 1990: Interessen, Ideen, Institutionen. Opladen: Westdeutscher Verlag.

Levi, M., 1996: A State of Trust. EUI-Working Paper RCS No. 96/23. European University Institute, Robert Schuman Center, Florenz.

Liebing, E., 1966: 75 Jahre Landesversicherungsanstalten. Die Deutsche Rentenversicherung: 68-82.
Mackenroth, G., 1952: Die Reform der Sozialpolitik durch einen deutschen Sozialplan. S. 39-89 in Albrecht, G. (Hrsg.), Verhandlungen auf der Sondertagung in Berlin, 18. und 19. April 1952. Schriften des Vereins für Socialpolitik NF, Bd. 4. Berlin: Duncker \& Humblot.

Maier, C.S., 1987: The Politics of Time: Changing Paradigms of Collective and Private Time in the Modern Era. S.151-175 in Maier, C.S. (Hrsg.), Changing Boundaries of the Political. Essays on the Evolving Balance between the State and Society, Public and Private in Europe. Cambridge: Cambridge University Press.

Majone, G., 1996: Temporal Consistency and Policy Credibility: Why Democracies need Non-Majoritarian Institutions. EUI-Working Paper, RSC No. 96/57. Badia Fiesolana, San Domenico (FI): European University Institute, Florence.

Manow, P., 1997: Entwicklungslinien ost- und westdeutscher Gesundheitspolitik zwischen doppelter Staatsgründung, deutscher Einigung und europäischer Integration. Zeitschrift für Sozialreform 43(2): $101-131$.

Manow, P., 1997: Social Insurance and the German Political Economy. MPIfG Discussion Paper 2/97. MaxPlanck Institut für Gesellschaftsforschung, Köln.

Moe, T.M., 1990: Political Institutions: The Neglected Side of the Story. Journal of Law, Economics, and Organization 6. Special Issue 1990: 213-253.

Mörschel, R., 1978: Die Zuschüsse des Staates zu den gesetzlichen Rentenversicherungen der Arbeiter und Angestellten. Die Deutsche Rentenversicherung: 332349.

Mörschel, R., 1990: Die Finanzierungsverfahren in der Geschichte der gesetzlichen Rentenversicherung. Die Deutsche Rentenversicherung: 619-661.

Nullmeier, F./Rüb, F.W., 1993: Die Transformation der Sozialpolitik. Vom Sozialstaat zum Sicherungsstaat. Frankfurt a.M.: Campus.

Offe, C., 1996: Designing Institutions in East European Transitions. S. 199-226 in Goodin, R.E. (Hrsg.), The Theory of Institutional Design. Cambridge: Cambridge University Press.

Orren, K./Skowronek, S., 1994: Beyond the Iconography of Order: Notes for a ,New Institutionalism'. S. 311-330 in Dodd, L.C./Jillson, C. (Hrsg.), The Dynamics of American Politics. Approaches and Interpretations. Boulder: Westview.

Orren, K./Skowronek, S., 1995: Order and Time in Institutional Study: A Brief for the Historical Approach. S. 296-356 in Farr, J./Dryzek, J.S./Leonard, S.T. (Hrsg.), Political Science in History. Research Programs and Political Traditions. Cambridge: Cambridge University Press.

Pierson, P., 1992: "Policy Feedbacks « and Political Change: Contrasting Reagan's and Thatcher's Pension-Reform Policies. Studies in American Political Development 6: 359-390.

Pierson, P., 1997: Increasing Returns, Path Dependence and the Study of Politics. Cambridge, MA (CES, Harvard University): unv. Manuskript. 
Powell, W.W./DiMaggio, P.J. (Hrsg.), 1991: The New Institutionalism in Organizational Analysis. Chicago/London: The University of Chicago Press.

Przeworski, A., 1988: Democracy as a Contingent Outcome of Conflicts. S. 59-80 in Elster, J./Slagstad, R. (Hrsg.), Constitutionalisms and Democracy. Cambridge: Cambridge University Press.

Przeworski, A., 1991: Democracy and the Market. Political and Economic Reforms in Eastern Europe and Latin America. Cambridge: Cambridge University Press.

Quellensammlung zur Geschichte der Deutschen Sozialpolitik [Quellensammlung], 1995 (II. Abteilung): Von der Kaiserlichen Sozialbotschaft bis zu den Februar erlassen Wilhelms II. (1881-1890). 2. Band, 1. Teil: Von der Zweiten Unfallversicherungs-Vorlage bis zum Unfallversicherungsgesetz vom 6.Juli 1884 . Bearbeitet von Florian Tennstedt und Heidi Winter. Stuttgart: Gustav Fischer.

Rosenstock, G., 1934: Versicherungstechnische Probleme in der Geschichte der Bismarck'schen Sozialgesetzgebung. Königsberg: Dissertation.

Rosin, H., 1914: Prämiendurchschnittsverfahren und Anwartschaftsdeckung. Monatsschrift für Arbeiter- und Angestellten-Versicherung 2: 97-106.

Roth, K.H., 1993: Intelligenz und Sozialpolitik im $»$ Dritten Reich火. Eine methodisch-historische Studie am Beispiel des Arbeitswissenschaftlichen Instituts der Deutschen Arbeitsfront. München: K.G. Saur.

Ruland, F., 1988: Anmerkungen zu den Vorschlägen von Schwarz-Schilling zur Rentenreform. Die Deutsche Rentenversicherung: 109-113.

Rürup, B., 1995: Renten- und Pensionsfinanzierung nach dem Kapitalstockverfahren - Möglichkeiten und Probleme. Die Deutsche Rentenversicherung: 718-725.

Scheur, W., 1967: Einrichtungen und Maßnahmen der sozialen Sicherheit in der Zeit des Nationalsozialismus. Dissertation, Köln.

Schewe, D., 1996: Der Satz vom Sozialaufwand aus dem gleichzeitigen Sozialprodukt. Herkunft und Zukunftsfragen. Sozialer Fortschritt 2/1996: 35-38.
Schimank, U., 1992: Erwartungssicherheit und Zielverfolgung. Sozialität zwischen Prisonerś Dilemma und Battle of the Sexes. Soziale Welt 43: 182-200.

Schmähl, W., 1981: Über den Satz „Aller Sozialaufwand muß immer aus dem Volkseinkommen der laufenden Periode gedeckt werden". Hamburger Jahrbuch für Wirtschafts- und Gesellschaftspolitik 26: 147-171.

Schmidt, M.G., 1990: Die Politik des mittleren Weges. Besonderheiten der Staatstätigkeit in der Bundesrepublik Deutschland. Aus Politik und Zeitgeschichte B 9-10/ 90: 23-31.

Schmitt, C., 1985: Der Hüter der Verfassung. (Dritte Auflage, unveränderter Nachdruck der 1931 erschienenen ersten Auflage). Berlin: Duncker \& Humblot.

Skowronek, S., 1997: The Politics Presidents Make. Cambridge, MA: Harvard University Press.

Stamm, A., 1972: Wachstumsorientierte Renten im Blickfeld der öffentlichen Verwaltung. S. 395 ff. in o.V., Demokratie und Verwaltung. 25 Jahre Hochschule für Verwaltungswissenschaften Speyer. Berlin: Duncker \& Humblot.

Stober, R., 1982: Verfassungsrechtlicher Eigentumsschutz sozialer Rechtspositionen. Schriftenreihe des Deutschen Sozialrechtsverbandes. Band XXIII, S.997. Wiesbaden.

Stolleis, M., 1984: Referat, sozialrechtliche Abteilung. S. N9-N68 in o.V., Verhandlungen des 55. Deutschen Juristentages. München: Beck.

Tennstedt, F./Winter, H., 1995: „Jeder Tag hat seine eigenen Sorgen, und es ist nicht weise, die Sorgen der $\mathrm{Zu}$ kunft freiwillig auf die Gegenwart zu übernehmen “ (Bismarck). Die Anfänge des Sozialstaats im Deutschen Reich von 1871. Teil 2.Zeitschrift für Sozialreform 41: 671-706.

Teppe, K., 1977: Zur Sozialpolitik des Dritten Reiches am Beispiel der Sozialversicherung. Archiv für Sozialgeschichte 17: 195-250.

Thullen, P., 1982: Das Prinzip der Beitragsäquivalenz im Zusammenhang mit den Finanzierungsverfahren der gesetzlichen Rentenversicherung. Die Deutsche Rentenversicherung: 124-144. 